\title{
Molecular Alterations in a Mouse Cardiac Model of Friedreich Ataxia
}

\section{An Impaired Nrf2 Response Mediated via Upregulation of Keap1 and Activation of the Gsk3 $\beta$ Axis}

\author{
Amy Anzovino, ${ }^{*}$ Shannon Chiang, ${ }^{*}$ Bronwyn E. Brown, ${ }^{\dagger \dagger}$ Clare L. Hawkins, ${ }^{\dagger \dagger \delta}$ Des R. Richardson, ${ }^{*}$ and Michael L.-H. Huang ${ }^{*}$
}

From the Molecular Pharmacology and Pathology Program, * Department of Pathology and Bosch Institute, and the Sydney Medical School, ${ }^{\ddagger}$ University of Sydney, Sydney, New South Wales, Australia; the Inflammation Group, ${ }^{\dagger}$ Heart Research Institute, Newtown, New South Wales, Australia; and the Department of Biomedical Sciences, ${ }^{\S}$ University of Copenhagen, Copenhagen, Denmark

Accepted for publication

August 17, 2017.

Address correspondence to Des R. Richardson, Ph.D., D.Sc., or Michael L.-H. Huang, Ph.D., Department of Pathology and Bosch Institute, University of Sydney, Medical Foundation Building K25, Sydney,

NSW, 2050, Australia. E-mail: d.richardson@sydney.edu.au or michael.huang@sydney.edu.au.

\begin{abstract}
Nuclear factor-erythroid 2-related factor-2 (Nrf2) is a master regulator of the antioxidant response. However, studies in models of Friedreich ataxia, a neurodegenerative and cardiodegenerative disease associated with oxidative stress, reported decreased Nrf2 expression attributable to unknown mechanisms. Using a mouse conditional frataxin knockout (KO) model in the heart and skeletal muscle, we examined the Nrf2 pathway in these tissues. Frataxin KO results in fatal cardiomyopathy, whereas skeletal muscle was asymptomatic. In the $\mathrm{KO}$ heart, protein oxidation and a decreased glutathione/ oxidized glutathione ratio were observed, but the opposite was found in skeletal muscle. Decreased total and nuclear Nrf2 and increased levels of its inhibitor, Kelch-like ECH-associated protein 1, were evident in the $\mathrm{KO}$ heart, but not in skeletal muscle. Moreover, a mechanism involving activation of the nuclear Nrf2 export/degradation machinery via glycogen synthase kinase-3 $\beta$ (Gsk3 $\beta$ ) signaling was demonstrated in the KO heart. This process involved the following: i) increased Gsk3 $\beta$ activation, ii) $\beta$-transducin repeat containing E3 ubiquitin protein ligase nuclear accumulation, and iii) Fyn phosphorylation. A corresponding decrease in Nrf2-DNA-binding activity and a general decrease in Nrf2-target mRNA were observed in KO hearts. Paradoxically, protein levels of some Nrf2 antioxidant targets were significantly increased in $\mathrm{KO}$ mice. Collectively, cardiac frataxin deficiency reduces $\mathrm{Nrf2}$ levels via two potential mechanisms: increased levels of cytosolic Kelch-like ECH-associated protein 1 and activation of Gsk3 $\beta$ signaling, which decreases nuclear Nrf2. These findings are in contrast to the frataxin-deficient skeletal muscle, where Nrf2 was not decreased. (Am J Pathol 2017, 187: 2858-2875; https://doi.org/10.1016/j.ajpath.2017.08.021)
\end{abstract}

Friedreich ataxia (FA) is a cardiodegenerative and neurodegenerative disorder caused by reduced expression of the mitochondrial protein, frataxin. ${ }^{1,2}$ In the heart, the loss of frataxin results in a fatal cardiomyopathy, leading to premature patient death. ${ }^{3}$ Surprisingly, little is known about the molecular dysfunction in FA, particularly in the heart, although it is usually ascribed to mitochondrial dysfunction. ${ }^{4}$ Electron micrographs of cardiac tissue from FA patients and from frataxin knockout (KO) mice demonstrate mitochondrial proliferation, loss of contractile sarcomeres, and distinct iron deposits within the mitochondria. ${ }^{5-7}$ It is hypothesized that the iron deposits within the highly oxidation-reduction (redox)-active environment of the mitochondrion can generate hydroxyl radicals through the Fenton reaction and cause oxidative stress. ${ }^{8}$ In fact, these

Supported by the National Health and Medical Research Council of Australia (NHMRC) Senior Principal Research Fellowship APP1062607 (D.R.R.) and project grant APP1128152 (D.R.R.), NHMRC Peter Doherty Early Career Fellowship APP1074033 (M.L.-H.H.), and Muscular Dystrophy Association development grant MDA417129 (M.L.-H.H.).

M.L.-H.H. and D.R.R. contributed equally to this work as senior authors. Disclosures: None declared. 
mitochondrial iron accumulations are not sequestered within mitochondrial ferritin and appear as inorganic crystallites that have the potential to participate in reactive oxygen species (ROS) generation. ${ }^{6}$

Oxidative stress is well described in FA, ${ }^{9,10}$ with evidence of hydroxyl radical formation, oxidative damage to DNA, and lipid peroxidation markers being identified in patient blood and urine samples. ${ }^{9,11}$ Furthermore, histology of FA patient spinal cord samples demonstrates increased glutathionylation, a protein modification caused by oxidative insult. ${ }^{10}$ In cell culture, FA patient fibroblasts exhibit increased oxidative modifications on treatment with ferrous salts, causing impaired cytoskeletal protein function and increased sensitivity to oxidative stress. ${ }^{12}$ When challenged with low doses of hydrogen peroxide or oligomycin, FA patient fibroblasts were unable to induce superoxide dismutase (SOD) activity, resulting in higher cell lethality compared with healthy controls. ${ }^{13}$ However, in these latter studies, the reason for the failed induction of SOD was not addressed. Oxidant-inactivating enzymes, such as SOD, and intracellular antioxidants, including glutathione (GSH) and thioredoxin, are the primary defense mechanisms of eukaryotic cells against ROS. ${ }^{14}$ However, perturbed antioxidant defense in the myocardium has not been investigated in the development of the fatal cardiomyopathy in FA.

The primary form of regulation of antioxidant defense occurs via de novo gene transcription. ${ }^{14}$ Regulation of a broad range of antioxidant genes is mediated by a consensus sequence located in the promoter region, known as the antioxidant response element (ARE) ${ }^{15,16}$ The AREs have been identified in hundreds of genes responsible for the detoxification of cellular ROS, collectively referred to as phase 2 (detoxifying and antioxidant protein) enzymes. The induction of the antioxidant response is controlled by the cap'n'collar bzip transcription factor, nuclear factorerythroid 2-related factor-2 (Nrf2). ${ }^{14,17}$ Two posttranslational mechanisms exist to regulate Nrf2 activity in both the cytosolic and nuclear compartments. These mechanisms are as follows: Kelch-like ECH-associated protein 1 (Keap1)-mediated sequestration of Nrf2 in the cytosol, which targets $\mathrm{Nrf} 2$ for proteasomal degradation; and glycogen synthase kinase-3 $\beta$ (Gsk3 $\beta$ )-dependent regulation of nuclear Nrf2, which leads to the phosphorylation, exportation, and degradation of nuclear Nrf2. ${ }^{18}$

Another level of antioxidant regulation comes from the transcriptional repressor, BTB domain and CNC homolog 1 (Bach1), which competes with $\mathrm{Nrf} 2$ for binding to AREs. ${ }^{19,20}$ In unstressed conditions, Bach1 is bound as a heterodimer with small Maf proteins to AREs, preventing the transcription of phase 2 genes. ${ }^{19,20}$ Interestingly, the activity and nuclear localization of Bach1 is heme regulated. ${ }^{21,22}$ When heme is bound to Bach1, its DNA-binding activity is decreased, which induces nuclear export of Bach1, reducing its repressive activity on the ARE. ${ }^{22}$ Considering that heme synthesis is markedly depressed in the heart of an FA mouse model, ${ }^{23}$ the expression and localization of Bach1 is important to assess. Notably, a decrease in Nrf2 expression has been noted in FA models, although the mechanism involved in reducing Nrf2 levels has not been deciphered. ${ }^{24,25}$

To examine Nrf2 function and redox homeostasis in FA cardiomyopathy, we examined the hearts from an established FA mouse model, the muscle creatine kinase (MCK) frataxin $\mathrm{KO}$ mouse. ${ }^{7,26}$ In these mice, the MCK promoterdriven CRE recombinase specifically excises frataxin in the striated muscle (ie, both cardiomyocytes and skeletal muscle cells). ${ }^{26}$ By 9 weeks of age, the KO mouse progressively develops a dilated cardiomyopathy, leading to heart failure, as in the human disease. ${ }^{7,26}$ Interestingly, and in marked contrast, despite the complete loss of frataxin, skeletal muscle pathology was not observed. ${ }^{26}$ The MCK $\mathrm{KO}$ mouse mimics the altered iron metabolism observed in FA patients, including the accumulation of mitochondrial iron in the heart, ${ }^{6,7,23,26}$ making it an excellent model to study FA pathogenesis.

In the current investigation, the MCK KO mouse was examined to assess redox stress and the antioxidant response. This study revealed significantly increased protein and GSH oxidation associated with ROS formation in the KO relative to wild-type (WT) littermates in the heart, but not in the skeletal muscle. Both total and nuclear Nrf2 expression was also found to be significantly decreased in the KO relative to the WT mouse heart. On investigation of well-characterized Nrf2 regulators, ${ }^{18}$ we demonstrated increased Keap1 expression and enhanced activation of nuclear export machinery via Gsk $3 \beta$ signaling, which are processes involved in decreasing cytosolic and nuclear Nrf2, respectively. These mechanisms would explain the observed decrease in nuclear Nrf2, Nrf2 ARE-binding activity, and the general decrease in the mRNA levels of antioxidant genes targeted by Nrf2 in the frataxin KO heart. In contrast to the heart, there were no significant alterations to the Nrf2 pathways in the frataxin KO skeletal muscle. Paradoxically, despite the general reduction in Nrf2 downstream antioxidant response genes at the mRNA level, their protein levels were either not reduced or significantly increased. This finding indicates that other pathways could at least partially compensate for the reduced Nrf2 levels and its transcriptional activity. Nonetheless, despite this compensation, oxidative damage still occurred in the heart, indicating the potential for antioxidant therapy for the treatment of this condition.

\section{Materials and Methods}

\section{Animals}

Transgenic C57B1/6 mice harboring MCK promoter-driven Cre recombinase expression that are homozygous for deletion of frataxin exon $4(\mathrm{KO})$ and their WT littermates were used and genotyped, as described previously. ${ }^{26}$ All animal 
work was approved by the University of Sydney's Animal Ethics Committee (Sydney, New South Wales, Australia).

\section{Histology}

MCK WT and KO mouse littermates were weighed and euthanized. The heart and skeletal muscle from the superficial part of the quadriceps were excised, freed from connective tissue and fat, washed in cold saline, blotted dry, weighed, and fixed in $10 \%$ formalin. Muscle samples were then cut and embedded in paraffin blocks, divided into sections, and stained with Perls' Prussian blue, Gömöri trichrome, or hematoxylin and eosin. Muscle fiber size was measured by ImageJ version 1.50i (NIH, Baltimore, MD; http://imagej.nih.gov/ij). ${ }^{27}$ Measurements were taken from at least three images from different animals (five WT and six $\mathrm{KO}$ ), and there were 10 measurements per image.

\section{Assessment of Protein 0xidation by HPLC}

Measurement of tyrosine oxidation products as a marker of protein oxidation in MCK mouse heart and skeletal muscle was performed by high-performance liquid chromatography (HPLC), as performed previously. ${ }^{28}$ Briefly, whole heart or skeletal muscle (left and right quadriceps) from WT and KO MCK mice was perfused with phosphate-buffered saline (pH 7.4) to thoroughly remove blood and then immediately snap frozen in liquid nitrogen. Frozen tissue samples were then ground to a fine powder using a mortar and pestle cooled with liquid nitrogen. Samples were resuspended in phosphate-buffered saline before precipitation of the proteins with trichloroacetic acid (10\% w/v) and acid hydrolysis of the resulting protein pellets with hydrochloric and thioglycolic acid under vacuum overnight at $110^{\circ} \mathrm{C}$. Samples were then separated by HPLC, detected using a UVvisible and fluorescence detector, and analyzed against analytical standards, as described. ${ }^{28}$

\section{Glutathione Measurement}

Total, reduced, and oxidized GSH were measured using Caymans Glutathione Assay Kit (Cayman Chemical, Ann Arbor, MI), following the manufacturer's instructions. Briefly, WT and KO whole heart or skeletal muscle (left and right quadriceps) was perfused with phosphate-buffered saline ( $\mathrm{pH} 7.4)$ to remove blood before extraction. Homogenization of tissues was done on ice in $5 \mathrm{~mL}$ of cold MES buffer [containing $50 \mathrm{mmol} / \mathrm{L}$ 2-(N-morpholino)ethanesulphonic acid (pH 6.0) and $1 \mathrm{mmol} / \mathrm{L}$ EDTA] using a motorized homogenizer. Samples were then centrifuged at $10,000 \times g / 15$ minutes $/ 4^{\circ} \mathrm{C}$, and the supernatant was removed for deproteination, as follows. Fresh metaphosphoric acid (Sigma-Aldrich, St. Louis, MO) was prepared and added in equal volume to the sample and vortex mixed. Samples were then incubated at room temperature for 5 minutes and centrifuged at $1500 \times g / 2$ minutes $/ 4^{\circ} \mathrm{C}$. The supernatant was collected, and freshly prepared $4 \mathrm{~mol} / \mathrm{L}$ triethanolamine (Sigma-Aldrich) was added to the samples and mixed by vortex mixing. Samples were then diluted 1:20 with MES buffer before performing the assay. A plate reader was used to measure the absorbance, which was read at $405 \mathrm{~nm}$ at 5-minute intervals for 30 minutes. GSH and oxidized GSH (GSSG) levels were expressed as a ratio.

\section{Protein Extraction}

Whole hearts and skeletal muscle (left and right quadriceps) from WT and KO MCK mice were perfused with phosphate-buffered saline ( $\mathrm{pH}$ 7.4) to remove excess blood before removal. Tissue was homogenized using a Dounce glass homogenizer in lysis buffer $[150 \mathrm{mmol} / \mathrm{L} \mathrm{NaCl}, 10$ $\mathrm{mmol} / \mathrm{L}$ Tris- $\mathrm{HCl}(\mathrm{pH} 7.4), 0.5 \%(\mathrm{w} / \mathrm{v}) \mathrm{SDS}, 1 \mathrm{mmol} / \mathrm{L}$ EDTA, $40 \mu \mathrm{mol} / \mathrm{L} \mathrm{NaF}$, and 1\% (v/v) Triton X-100] containing a $1 \times$ solution of PhosSTOP (Roche Diagnostics, Risch-Rotkreuz, Switzerland) and a $1 \times$ solution of protease inhibitor cocktail (Roche Diagnostics). Lysates were then sonicated on ice and centrifuged at 13,200 $\times$ g/40 minutes/ $4^{\circ} \mathrm{C}$. The supernatant was collected, and the protein concentration was determined by the BCA Protein Assay (Pierce Biotechnology, Rockford, IL).

\section{Nuclear and Cytosolic Fractions}

Cytosolic and nuclear fractions were prepared from MCK whole hearts and skeletal muscle (left and right quadriceps) using NE-PER nuclear and cytosolic extraction reagents (Thermo Fisher Scientific, Waltham, MA). Fractionation was performed according to the kit instructions. Tissue samples were first homogenized in a Dounce glass homogenizer. Tissue lysates were then supplemented with a $1 \times$ solution of protease inhibitor (Roche Diagnostics) and $1 \times$ solution of PhosSTOP.

\section{Protein Separation and Western Blot Analysis}

Protein lysates were heat denatured at $95^{\circ} \mathrm{C} / 2$ minutes in the presence of $\beta$-mercaptoethanol. Then, $50 \mu \mathrm{g}$ of protein or molecular weight marker (Bio-Rad, Hercules, CA) was loaded onto an $8 \%$ to $12 \%$ polyacrylamide gel and separated using SDS-PAGE. Proteins were then transferred overnight $\left(30 \mathrm{~V} / 4^{\circ} \mathrm{C}\right.$ ) onto a polyvinylidene difluoride membrane (pore size, 0.2 or $0.45 \mu \mathrm{m}$; Millipore, Billerica, MA). Membranes were then blocked for 1 hour at room temperature in either 5\% skim milk prepared in Tris-buffered saline and $0.1 \%$ Tween- 20 or $5 \%$ bovine serum albumin/Trisbuffered saline and $0.1 \%$ Tween-20. Primary antibodies were diluted in either 5\% skim milk/Tris-buffered saline and $0.1 \%$ Tween- 20 or $5 \%$ bovine serum albumin/Tris-buffered saline and $0.1 \%$ Tween-20, added to membranes, and incubated overnight at $4^{\circ} \mathrm{C}$ on a rocker. Antibodies used were against Nrf2 (Santa Cruz Biotechnology, Dallas, TX; 
catalog number sc-722), Bach1 (Santa Cruz Biotechnology; catalog number sc-14700), Keap1 (ProteinTech, Rosemont, IL; catalog number 10503-2-AP), phosphorylated Gsk3 $\beta$ serine 9 (Cell Signaling, Danvers, MA; catalog number 9332), tyrosine 216 (Abcam, Cambridge, MA; catalog number ab75745), Gsk3 $\beta$ (Cell Signaling; catalog number 9832), phosphorylated Fyn threonine 12 (Santa Cruz Biotechnology; catalog number sc-16848), Fyn (Cell Signaling; catalog number 40238), phosphorylated tyrosine (Cell Signaling; catalog number 9416), $\beta$-transducin repeat containing E3 ubiquitin protein ligase $(\beta-\mathrm{TrCP}$; Cell Signaling; catalog number 11984), glyceraldehyde-3phosphate dehydrogenase (Gapdh; Cell Signaling; catalog number 2118), and histone deacetylase-1 (Santa Cruz Biotechnology; catalog number sc-8410). Antibodies were used at 1:500 to 1:2000. Secondary antibodies used were goat anti-rabbit, rabbit anti-goat, and goat anti-mouse (A0545, A5420, and A9917, respectively; 1:10,000; Sigma-Aldrich) conjugated with horseradish peroxidase.

\section{Immunoprecipitation}

Whole hearts were homogenized in $500 \mu \mathrm{L}$ of ice-cold radioimmunoprecipitation assay (RIPA) buffer $[50 \mathrm{mmol} /$ L Tris (pH 7.5), $150 \mathrm{mmol} / \mathrm{L} \mathrm{NaCl}, 1 \mathrm{mmol} / \mathrm{L}$ EDTA, $1 \%$ Nonidet P-40, 0.5\% (w/v) sodium deoxycholate, $0.1 \%$ (w/v) SDS, and $1 \times$ solution of protease and phosphatase inhibitors (Roche Diagnostics)] using a Dounce glass homogenizer. The resulting lysate was incubated for 30 minutes $/ 4^{\circ} \mathrm{C}$ plus rotation to ensure complete lyses of cells and then centrifuged at $13,200 \times g / 40$ minutes $/ 4^{\circ} \mathrm{C}$. The supernatant was collected in a new sterile Eppendorf tube, and the protein concentration was determined using the BCA Protein Assay. Then, $30 \mu \mathrm{L}$ of Dynabeads protein $\mathrm{G}$ (Novex; Thermo Fisher Scientific) per sample was placed in Eppendorf tubes and washed twice with RIPA buffer. After the second wash, the beads were resuspended in 1 $\mathrm{mL}$ of RIPA buffer, and $4 \mu \mathrm{g}$ of Nrf2 antibody (Cell Signaling; catalog number 12721) per tube was added. The primary antibody was conjugated to the beads after incubation for 2 hours $/ 4^{\circ} \mathrm{C}$. After incubation, the conjugated beads were collected on a magnet, and the supernatant was removed. The conjugated beads were washed twice with ice-cold RIPA buffer, and the last wash was discarded.

Then, $500 \mu \mathrm{g}$ of protein lysate was added per tube of conjugated beads and the volume was increased to $1 \mathrm{~mL}$. Lysates and antibody-conjugated beads were incubated at $4^{\circ} \mathrm{C}$ plus rotation overnight. The next day, the beads were collected on a magnet, and the supernatant was discarded. The beads were washed 3 times with ice-cold RIPA buffer and then resuspended in $35 \mu \mathrm{L}$ of $1 \times$ loading dye plus $\beta$-mercaptoethanol. The samples were incubated at 5 minutes $/ 95^{\circ} \mathrm{C}$ to disassociate the complexes from the beads. The beads were then collected on the magnet, the supernatant was separated on a $10 \%$ polyacrylamide gel, and Western blot analysis was performed, as described above.

\section{Electrophoretic Mobility Shift Assay}

Electrophoretic mobility shift assay reactions were conducted in $20 \mathrm{mmol} / \mathrm{L}$ HEPES (pH 7.9) containing $1 \mathrm{mmol} / \mathrm{L}$ EDTA, $50 \mathrm{mmol} / \mathrm{L} \mathrm{KCl}, 5 \mathrm{mmol} / \mathrm{L} \mathrm{MgCl}_{2}, 4 \%$ (v/v) glycerol, $1 \mathrm{mmol} / \mathrm{L}$ dithiothreitol, $3 \mu \mathrm{g} / \mathrm{mL}$ poly $(\mathrm{dI}-\mathrm{dC})$, 36-bp end-labeled quinone 1 ( $\mathrm{Nqol}$ ) ARE sequence (5'AGTCTAGAGTCACAGTGAGTGCCAAAATTTGAGCC$3^{\prime}$, corresponding to nucleotides -451 to -416 ), and $20 \mu \mathrm{g}$ of nuclear protein. In competition experiments, a 200 -fold molar excess of unlabeled 36-bp Nqol ARE sequence was also included in the reaction. Reactions were incubated for 30 minutes $/ 20^{\circ} \mathrm{C}$ before being subjected to electrophoresis under native conditions using a $6 \%(\mathrm{w} / \mathrm{v})$ polyacrylamide $(1: 75$ bisacrylamide/acrylamide) gel in $0.5 \times$ Tris/Borate/EDTA buffer at $4^{\circ} \mathrm{C}$. Gels were then transferred onto Immobilon$\mathrm{Ny}+$ membrane and detected by Chemiluminescent Nucleic Acid Detection Module Kit (Thermo Fisher Scientific; catalog number 89880).

\section{RNA Isolation from Tissue}

Whole hearts from WT and KO MCK mice were homogenized using a motorized homogenizer. Homogenizer probes had been treated in diethyl pyrocarbonate water overnight and sterilized before use. RNA extraction was done using TriReagent, following manufacturer's protocol (Invitrogen, Carlsbad, CA), and quantified using a NanoDrop spectrophotometer (Thermo Fisher Scientific).

\section{Real-Time Quantitative RT-PCR}

Total mRNA was obtained from whole hearts and used for cDNA synthesis using a High Capacity cDNA Reverse Transcription Kit (Thermo Fisher Scientific). Quantitative PCR was performed using the Roche Lightcycler 480 (Roche Diagnostics). TaqMan probes (Thermo Fisher Scientific) targeting mouse Nrf2 (Mm00477784_m1), Nqo1 (Mm01253561_m1), thioredoxin reductase 1 (TxnRD1; Mm00443675_m1), glutatione-S-transferase Mu1 (Gstm1; Mm00833915_g1), Sod2 (Mm01313000_m1), catalase (Mm00437992_m1), and Gapdh (Mm99999915_g1) were used. Standard curves were generated for each probe, and samples were fitted to the linear portion of the curve. Data were analyzed using Genex Software version 6.1 (MultiD Analyses, Göteborg, Sweden).

\section{Statistical Analysis}

Data were compared using $t$-test. Data were considered statistically significant when $P<0.05$. Results are expressed as means \pm SEM. 


\section{Results}

Identification of Oxidative Stress in the FrataxinDeficient Heart, but Not the Skeletal Muscle

Oxidative stress is hypothesized to play a role in the pathogenesis of FA. ${ }^{9,13,25}$ Previous examination of the iron deposits in the cardiac mitochondria of 9-week-old MCK KO mice revealed that they were distinct from the iron bound by the iron storage protein ferritin. ${ }^{6}$ In fact, this mitochondrial iron was demonstrated to be in the form of an inorganic iron crystallite that could potentially be redox active. ${ }^{6}$ This is particularly significant, because non-ferritin-bound iron can result in cytotoxic ROS generation. ${ }^{8}$

In the current investigation, we assessed the MCK KO mouse, which specifically exhibits deletion of frataxin within striated muscle (namely, the heart and skeletal muscle). ${ }^{7,26}$ Despite frataxin deletion in both tissues, it has been reported that there is a remarkable difference in terms of the pathology observed, ${ }^{26}$ and the expression of proteins involved in cellular iron metabolism. ${ }^{6}$ However, there has been no direct comparison of the histology between the heart and skeletal muscle, their relative redox stress status, or related molecular alterations. Hence, these aspects were examined herein.

Initial studies assessed the gross histological alterations in the heart and skeletal muscle of the MCK WT and KO mice at 9 weeks of age, when there is a pronounced phenotype of cardiac hypertrophy. ${ }^{7,26}$ Using Perls' Prussian blue, Gömöri trichrome, and hematoxylin and eosin staining, the 9-week-old MCK KO heart demonstrated the following: i) myofiber hypertrophy with iron accumulation (Figure 1, $\mathrm{A}$ and D); ii) interstitial fibrosis (Figure 1, B and E); and iii) myofibrillar disarray (Figure 1, C and F). These findings were relative to the WT littermates, as reported previously. $^{7,26}$ In contrast, despite complete frataxin loss, ${ }^{7,26}$ the quadriceps skeletal muscle in the 9-week-old MCK KO mouse showed no histopathological alterations relative to the WT mice (Figure 1, G-L), ${ }^{26}$ as found for FA patients. $^{29,30}$ Notably, although the cross-sectional area of the muscle fibers was found to be significantly $(P<0.001)$ greater in the heart muscle of the KO mice $\left(562 \pm 42 \mu \mathrm{m}^{2}\right)$ relative to WT mice $\left(310 \pm 30 \mu \mathrm{m}^{2}\right)$, the skeletal muscle of KO mice was significantly $(P<0.001)$ smaller $(880 \pm 98$ $\left.\mu \mathrm{m}^{2}\right)$ compared with their WT littermates $\left(2057 \pm 190 \mu \mathrm{m}^{2}\right)$ (Figure 1M). However, as demonstrated previously, the MCK KO mouse markedly loses body weight relative to the WT control. ${ }^{26,31}$ As a consequence, the heart/body weight ratio was significantly $(P<0.001)$ higher in the $\mathrm{KO}(1.27 \%$ $\pm 0.08 \% ; n=6)$ relative to the WT littermates $(0.54 \% \pm$ $0.01 \% ; n=4$ ) (Figure $1 \mathrm{~N}$ ). In contrast to the heart, the skeletal muscle (quadriceps)/body weight ratio was not significantly $(P>0.05)$ altered between the KO and WT mice (Figure 1N). This observation demonstrates that, although there was a marked reduction in skeletal muscle fiber area in $\mathrm{KO}$ relative to WT mice (Figure $1 \mathrm{M}$ ), this decrease was proportional to the loss of body weight in $\mathrm{KO}$ littermates (Figure 1N).

Considering these observations, we then assessed redox stress in the whole heart compared with quadriceps skeletal muscle from WT and KO littermates at 9 weeks of age (Figure 2). This was achieved by using HPLC, which is a sensitive method for the detection and quantification of protein oxidation products. ${ }^{28}$ In addition, our studies also examined the major intracellular antioxidant, GSH, and its oxidized counterpart, GSSG, via an established method. ${ }^{32}$

Whole hearts from 9-week-old MCK WT and KO mice were assayed by HPLC, and a significant $(P<0.01 ; n=12)$ increase in $o$-Tyr relative to Tyr was identified in the hearts of KO mice compared with WT mice (Figure 2A). This observation indicated increased protein oxidation that has been reported in other disease states and that could occur through generation of highly reactive hydroxyl radicals. ${ }^{33,34}$

We then examined the levels of the antioxidant, $\mathrm{GSH},{ }^{35}$ and demonstrated that in the heart of 9-week-old $\mathrm{KO}$ mice, relative to WT littermates, the following were found: i) a significant $(P<0.01)$ increase in total glutathione (ie, $\mathrm{GSH}+\mathrm{GSSG})($ Figure $2 \mathrm{C})$, ii) a significant $(P<0.01)$ increase in GSSG (Figure 2E), and iii) no significant change in GSH levels in the heart of $\mathrm{KO}$ mice (Figure 2G). These alterations in GSH resulted in a significant $(P<0.01)$ decrease in the ratio of GSH/GSSG in the heart of the MCK $\mathrm{KO}$ mice relative to their WT littermates, indicating increased GSH oxidation (Figure 2I).

In contrast to the oxidative stress in the heart, examination of skeletal muscle demonstrated a slight, nonsignificant $(P>$ 0.05 ) decrease in $o$-Tyr relative to Tyr in the $\mathrm{KO}$ relative to WT mice (Figure 2B). In terms of GSH status, in the skeletal muscle of $\mathrm{KO}$ mice relative to WT mice, we observed the following: i) a slight, but significant $(P<0.01)$, decrease in total glutathione (ie, GSH + GSSG) (Figure 2D); ii) a significant $(P<0.001)$ decrease in GSSG levels (Figure $2 \mathrm{~F}$ ); and iii) no significant $(P>0.05)$ change in GSH levels (Figure 2H). These changes in GSH and GSSG levels resulted in a significant $(P<0.05)$ increase in the GSH/ GSSG ratio (Figure $2 \mathbf{J}$ ) in the skeletal muscle of $\mathrm{KO}$ compared with WT mice. Collectively, these data in Figure 2 demonstrate that despite frataxin deficiency in both the heart and skeletal muscle of MCK KO mice, ${ }^{6,26}$ oxidative stress is evident in the heart, but not the skeletal muscle.

Decreased Total Nrf2 Expression Is Observed in the KO Heart and Corresponds with Increased Keap1 Expression

The so-called master regulator of antioxidant gene transcription, Nrf2, has become a focus in terms of understanding the cellular response to oxidative stress, with some recent reports of defective Nrf2 responses in multiple FA models, ${ }^{24,25,36}$ but not the heart or skeletal muscle. Indeed, previous studies in cellular models of FA have reported that a decrease in Nrf2 nuclear translocation and nuclear levels 


\section{Cardiac Histopathology at End-Stage (9 weeks)}

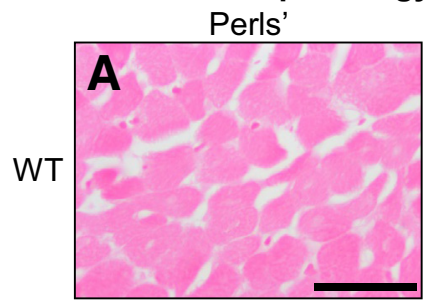

Gömöri Trichrome
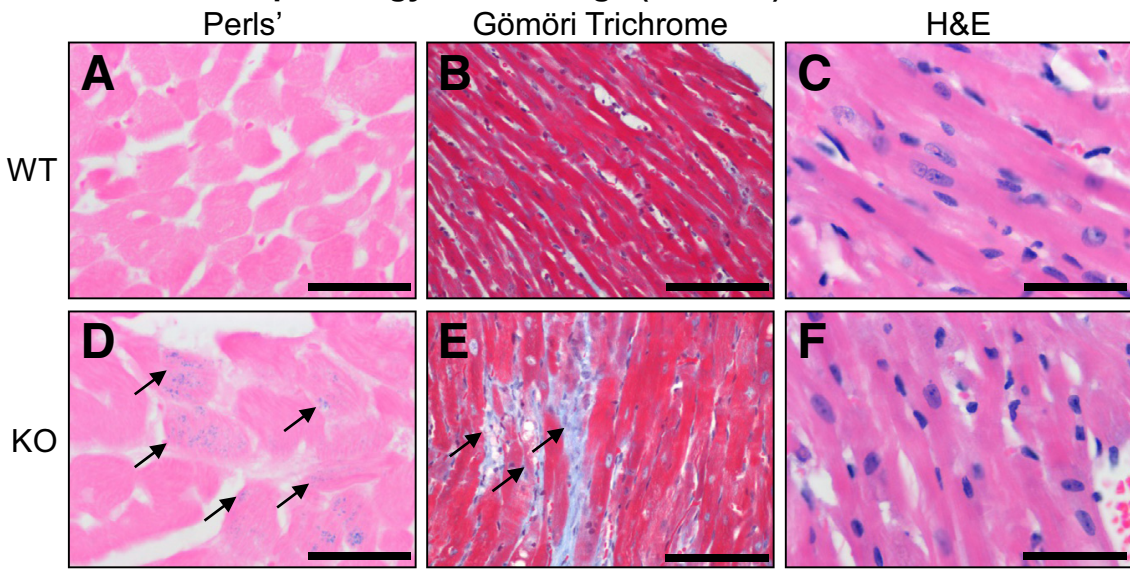

Skeletal Muscle Histopathology at End-Stage (9 weeks)

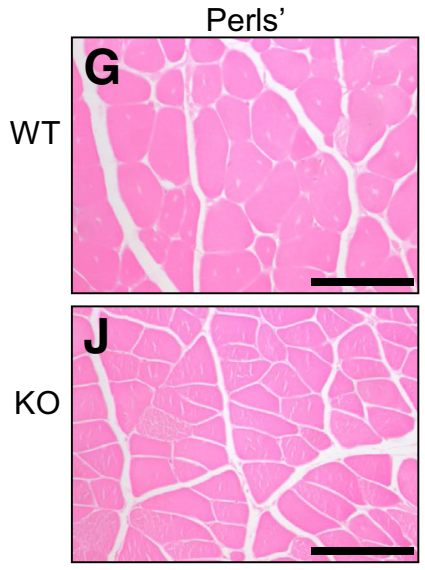

Gömöri Trichrome
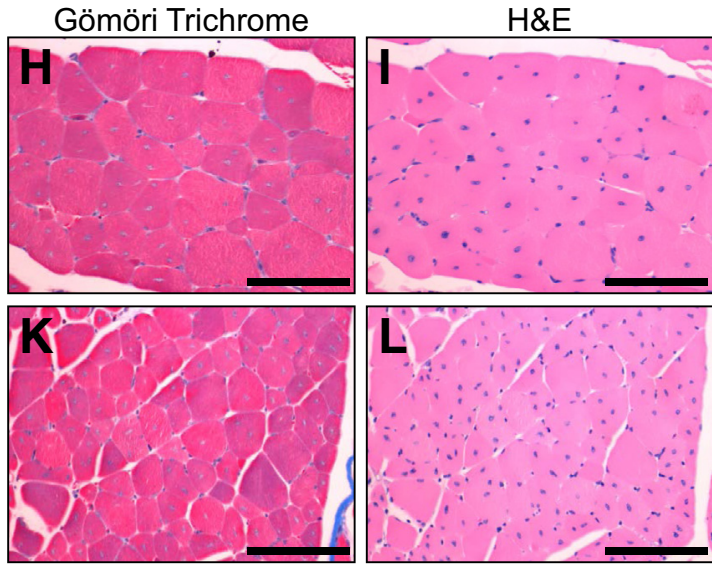

M

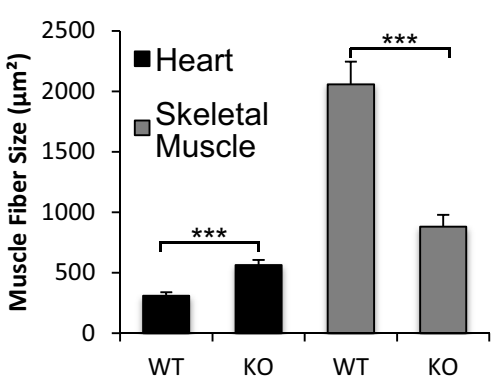

N

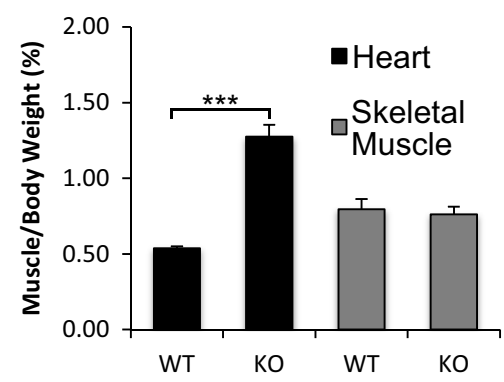

Figure 1 Pronounced histopathology of the heart, but not the skeletal muscle, of MCK knockout (KO) mice. A-L: Histological staining of heart $(\mathbf{A}-\mathbf{F})$ and skeletal muscle $(\mathbf{G}-\mathbf{L})$ from 9-week-old wild-type (WT; $\mathbf{A}-\mathbf{C}$ and $\mathbf{G}-\mathbf{I}$ ) and K0 (D-F and $\mathbf{J}-\mathbf{L}$ ) littermates. The onset of Friedreich ataxia cardiac histopathological features can be observed at 9 weeks of age, when the KO mice succumb to cardiomyopathy. Hearts and skeletal muscle were stained with Perls' Prussian blue (left column; A, D, G, and J), Gömöri trichrome (middle column; $\mathbf{B}, \mathbf{E}, \mathbf{H}$, and $\mathbf{K}$ ), and hematoxylin and eosin (H\&E; right column; C, F, I, and L). Arrows denote iron-positive cardiomyocytes (D) myocardial fibrosis, as depicted by the blue/gray staining (E). In the heart, $\mathbf{A}$ and $\mathbf{D}$ are transverse sections, whereas $\mathbf{B}, \mathbf{C}, \mathbf{E}$, and $\mathbf{F}$ are longitudinal sections. $\mathbf{G}-\mathbf{L}$ : In the skeletal muscle, all sections are transverse sections. Representative histological staining is shown. $\mathbf{M}$ and $\mathbf{N}$ : Analysis of muscle fiber size (M) and muscle/body weight $(\% ; \mathrm{N})$ between the heart and skeletal muscle of 9-week-old WT and KO littermates. Data are expressed as means \pm SEM. $n=5$ to 6 in each group ( $\mathrm{M}$ and $\mathrm{N}$ ). ${ }^{* * * P}<0.001$ versus WT. Scale bars: $40 \mu \mathrm{m}(\mathbf{A}$ and $\mathbf{D}-\mathbf{F}) ; 100$ $\mu \mathrm{m}(\mathbf{B}, \mathbf{C}$, and $\mathbf{G}-\mathbf{L})$. of this protein could be responsible for the blunted antioxidant response to frataxin deficiency. ${ }^{24,36}$ Considering these investigations and the results in Figure 2, our studies then examined if $\mathrm{Nrf} 2$ protein expression was affected in the heart relative to the skeletal muscle in MCK KO and WT mice (Figure 3).

In the heart, Western blot analysis using total protein lysates from 9-week-old MCK mice confirmed that frataxin expression was almost ablated in the $\mathrm{KO}$ compared with WT littermates (Figure 3A). Total ablation of cardiac frataxin was not observed, because the heart tissue sample is composed of a small proportion of fibroblasts, nerves, and endothelial cells, where the frataxin gene remains intact. Our studies showed, using total protein lysates, that Nrf2 expression in the $\mathrm{KO}$ heart was significantly $(P<0.001)$ decreased relative to the WT heart (Figure 3A). Similarly, the ARE transcriptional repressor, Bach1, which competes for ARE binding with $\mathrm{Nrf} 2,{ }^{19,20}$ was also significantly $(P<$ $0.01)$ decreased in the KO mice compared with WT littermates (Figure 3A). These data suggest the Bach1-mediated transcriptional repression of ARE-containing genes ${ }^{19}$ may not be as marked in the $\mathrm{KO}$ relative to the WT heart. Moreover, reduced Bach1 in the KO may be attributable to decreased Nrf2 levels, because Bach1 is positively regulated by $\mathrm{Nrf} 2$ as part of a feedback-inhibitory mechanism. ${ }^{37}$ In fact, Bachl has been reported to contain an ARE in its promoter region and is a transcriptional target of Nrf2. ${ }^{37}$

Because Keap1 regulates Nrf2 by binding to its $C$ terminal and sequestering it within the cytosol under physiological conditions, ${ }^{38}$ we examined Keap1 levels to 

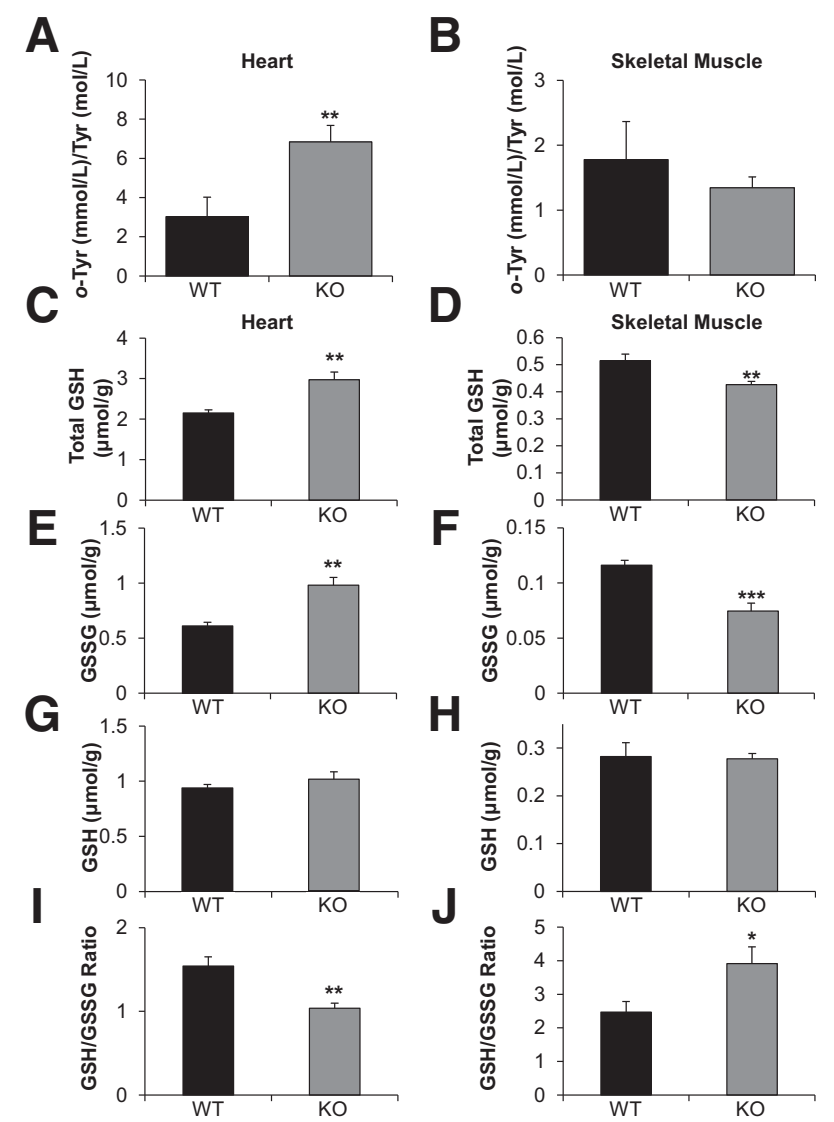

Figure 2 Increased levels of markers of oxidative stress in the heart, but not the skeletal muscle, of MCK frataxin wild-type (WT) and knockout (KO) mice. Heart (A, C, E, G, and I) and skeletal muscle (B, D, F, H, and $\mathbf{J})$ of the MCK frataxin KO mice relative to WT littermates were examined at 9 weeks of age for 0 -Tyr/Tyr ratio that indicates protein oxidation using highperformance liquid chromatography (A and $\mathbf{B})$ and glutathione (GSH; $\mathbf{C}-\mathbf{J}$ ) oxidation by examining total GSH (C and $\mathbf{D})$, oxidized GSH (GSSG; $\mathbf{E}$ and $\mathbf{F}$ ), GSH ( $\mathbf{G}$ and $\mathbf{H}$ ), and GSH/GSSG ratio ( $\mathbf{I}$ and $\mathbf{J})$. Values for total GSH, GSSG, and $\mathrm{GSH}$ were expressed as $\mu \mathrm{mol} / \mathrm{g}$ tissue. Data are means \pm SEM (A-J). $n=3$ experiments $(\mathbf{A}-\mathbf{J}) .{ }^{*} P<0.05,{ }^{*} P<<0.01$, and ${ }^{* *} P<0.001$ versus WT.

determine whether they could be a mechanism responsible for the observed decrease in total Nrf2 levels. Interestingly, Keap1 was markedly and significantly $(P<0.001)$ increased in the KO relative to the WT in these total heart lysates from 9-week-old mice (Figure 3A), suggesting Keap1 mediated degradation of Nrf2. ${ }^{18}$ Because cardiomyopathy and functional deficits are only apparent in the MCK KO mice from 7 weeks of age, ${ }^{7,26}$ studies also assessed alterations in Nrf2, Bach, and Keap1 expression in these mice at 4 weeks of age, when there is no gross phenotype. ${ }^{7,26}$ These studies demonstrated a slight, but not significant $(P>0.05)$, decrease in $\mathrm{Nrf} 2$ expression between 4-week-old KO and WT hearts (Supplemental Figure S1). In contrast, a significant $(P<0.001)$ decrease in Bach1 and a significant $(P<0.01)$ increase in Keap1 were observed in the KO relative to the WT hearts (Supplemental Figure S1). This increase in Keap1 may be responsible for the slight decrease in Nrf2. ${ }^{38}$ Hence, Keap1 appeared to be an early molecular marker of the dysfunctional Nrf2 pathway in $\mathrm{KO}$ mice.

As a relevant comparison to the heart, we also examined Nrf2, Bach1, and Keap1 expression in skeletal muscle from 9-week-old mice, because frataxin is also deleted in this tissue $^{26}$ (Figure 3B). When examining skeletal muscle, frataxin expression was confirmed to be markedly ablated in the $\mathrm{KO}$ relative to WT littermates (Figure 3B), as shown previously. ${ }^{6,26}$ Again, as discussed above for the heart, total ablation of frataxin could not be expected, because the total skeletal muscle lysate contains a small proportion of other cell types (eg, fibroblasts and endothelial cells) that do not harbor the frataxin deletion. ${ }^{7,26}$ In clear contrast to the heart of the MCK KO mouse (Figure 3A), there was no significant $(P>0.05)$ alteration of Nrf2, Bach1, or Keap1 expression between the MCK KO and WT littermates, despite the deficiency of frataxin expression (Figure 3B). No significant alteration in Nrf2 or Bach1 expression was observed in the skeletal muscle at 4 weeks of age (Supplemental Figure S1). On the other hand, a significant $(P<0.01)$ decrease in Keap1 was observed (Supplemental Figure S1). In summary, the alteration in Nrf2 expression appears to be tissue specific and was only observed in the 9week-old mouse heart (Figure 3A), where a pronounced phenotype is apparent. ${ }^{6,26}$

\section{Nuclear Nrf2 Expression Is Decreased in the 9-Week-0ld KO Heart}

Considering the following: i) the pro-oxidant environment in the $\mathrm{KO}$ heart (Figure 2, A, C, E, G, and I), ii) the decreased Nrf2 in the KO heart (Figure 3A), and iii) the reported impaired Nrf2 nuclear translocation in cellular FA models, ${ }^{24,36}$ we next examined the nuclear levels of Nrf2 in the KO versus WT heart (Figure 3C).

Nuclear and cytosolic fractions were prepared from MCK mouse hearts and examined by Western blot analysis for the subcellular distribution of Nrf2 (Figure 3C). In these studies, Gapdh (a cytosolic marker) and histone deacetylase-1 (a nuclear marker) were examined to ensure fraction identity and to assess the possibility of fraction cross-contamination (Figure 3C). The expression of Nrf2 was predominantly nuclear in the heart of both WT and KO mice, with Nrf2 nuclear expression being significantly $(P<0.01)$ reduced in the KO relative to the WT littermates (Figure 3C). Similarly, Bach1 was also significantly $(P<0.001)$ reduced in both the cytosolic and nuclear fractions of the KO relative to WT mice (Figure 3C). Conversely, Keap1 was predominantly cytosolic, as expected ${ }^{39}$ with a significant $(P<0.01$ to $P<0.05)$ increase in its expression in both cytosolic and nuclear fractions being evident in the KO heart, relative to their WT counterparts (Figure 3C). Considering the presence of nuclear Keap1, this observation can be explained by the following: the translocation of Keap1 into the nucleus, where it can complex with Nrf2 to facilitate Keap1-Nrf2 nuclear export and subsequent degradation ${ }^{40,41}$; and/or the slight crossover of 
the nuclear with the cytosolic fraction, as shown by marker analysis (Figure 3C).

Interestingly, cellular fractionation of skeletal muscle at 9 weeks of age demonstrated no significant $(P>0.05)$ alteration in the cytosolic or nuclear expression of Nrf2, Bach1, or Keap1 between WT and KO mice (Figure 3D). Despite harboring the same frataxin deficiency, ${ }^{7,26}$ this marked difference in Nrf2 pathway regulation between the heart and skeletal muscle demonstrates the clear tissuespecific effects of frataxin.

In summary, in the heart of MCK KO mice, there is a decrease in total Nrf2 expression that could be mediated by the increase in cytosolic Keap1. In contrast, despite the knockout of frataxin in the skeletal muscle, there was no significant $(P>0.05)$ alteration in the expression of Nrf2, Bach1, or Keap1. This tissue specificity is of considerable interest, because systemic frataxin deficiency in FA patients only causes a pathologic phenotype in certain tissues, with the heart being markedly affected. ${ }^{6,42}$
The Gsk3 $\beta$-Mediated Pathway that Decreases Nuclear Nrf2 Is Activated in the Heart of Frataxin KO Mice

In addition to the potential role of Keap1 in decreasing Nrf2 in the heart of MCK KO mice (Figure 3, A and C), Keap1independent mechanisms of Nrf2 down-regulation involve Nrf2 phosphorylation by Gsk3 $\beta$-dependent pathways that regulate Nrf2 nuclear localization. ${ }^{43-46}$ These mechanisms are examined in Figure 4, because they could also explain the decreased nuclear and total Nrf2 levels observed in the MCK KO heart.

First, our studies examined the phosphorylation status of Gsk $3 \beta$, which governs its kinase activity and ability to effect downstream targets, such as Nrf2. ${ }^{43-45}$ Both the inactivating (Ser9) and activating (Tyr216) phosphorylation sites of Gsk $3 \beta^{47}$ were examined by Western blot analysis in total cell lysates from MCK WT and KO hearts (Figure 4A). The pSer9/Gsk $3 \beta$ ratio was significantly $(P<0.05)$ decreased in the KO relative to WT hearts, whereas the pTyr216/Gsk $3 \beta$ ratio was significantly $(P<0.01)$ increased (Figure $4 \mathrm{~A})$. In
A
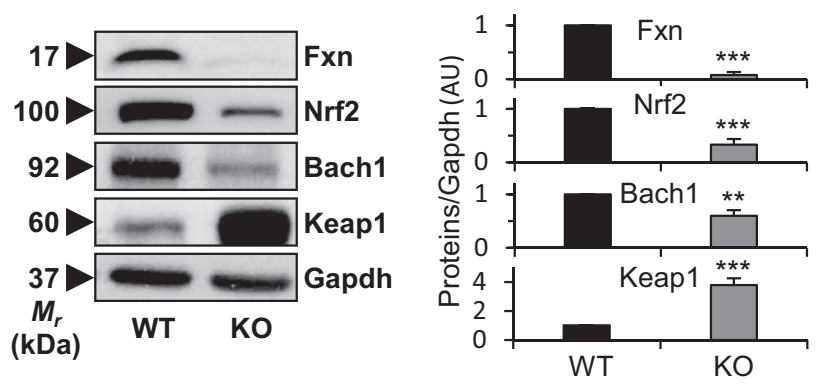

C

Heart Fractionation
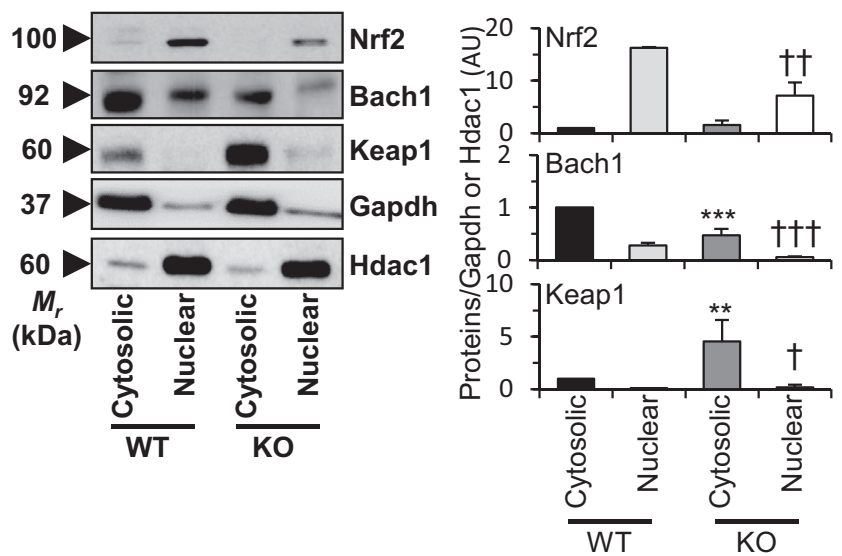

\section{B Skeletal Muscle (Total Protein)}
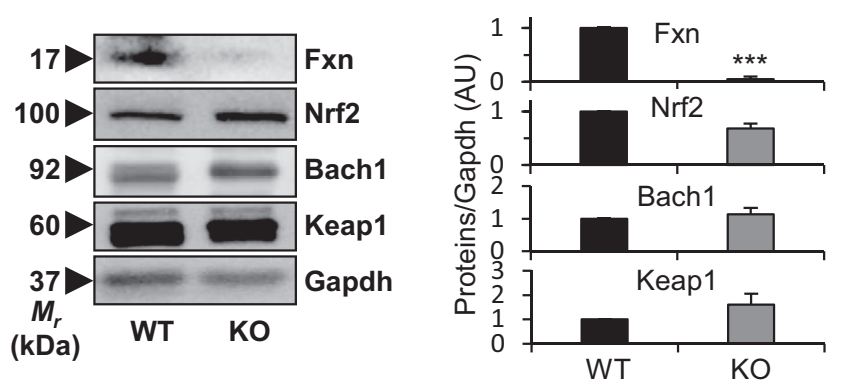

D Skeletal Muscle Fractionation
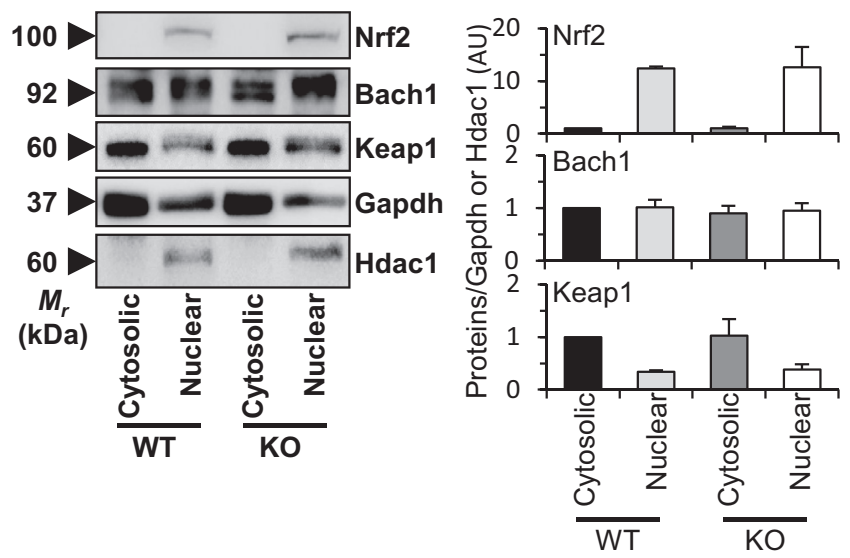

Figure 3 Western blot analysis showing alterations in expression of Nrf2, Bach1, and Keap1 in the heart, but not the skeletal muscle, of MCK frataxin knockout (KO) mice. Total protein (A and B) and cytosolic and nuclear lysates (C and D) from heart and skeletal muscle of the MCK frataxin (Fxn) wild-type (WT) and KO mice. The MCK frataxin KO mice relative to WT littermates were examined at 9 weeks of age. Western blot analysis of frataxin, Nrf2, Bach1, and Keap1 expression in the heart and skeletal muscle. A and B: Glyceraldehyde-3-phosphate dehydrogenase (Gapdh) was used as a protein-loading control and implemented for normalization of protein expression. C and D: Gapdh and histone deacetylase-1 (Hdac1) were used to assess the cytosolic and nuclear fractions, respectively, and implemented for normalization of protein loading. Western blot analysis shown is typical of three to four experiments. The densitometry data are means \pm SEM (A-D). $n=3$ to 4 experiments (A-D). ${ }^{* * P} P<0.01,{ }^{* * *} P<0.001$ versus WT or WT cytosolic; ${ }^{\dagger} P<0.05,{ }^{\dagger \dagger} P<0.01$, and ${ }^{\dagger \dagger \dagger} P<0.001$ versus WT nuclear. AU, arbitrary unit; $M_{\mathrm{r}}$, molecular mass. 

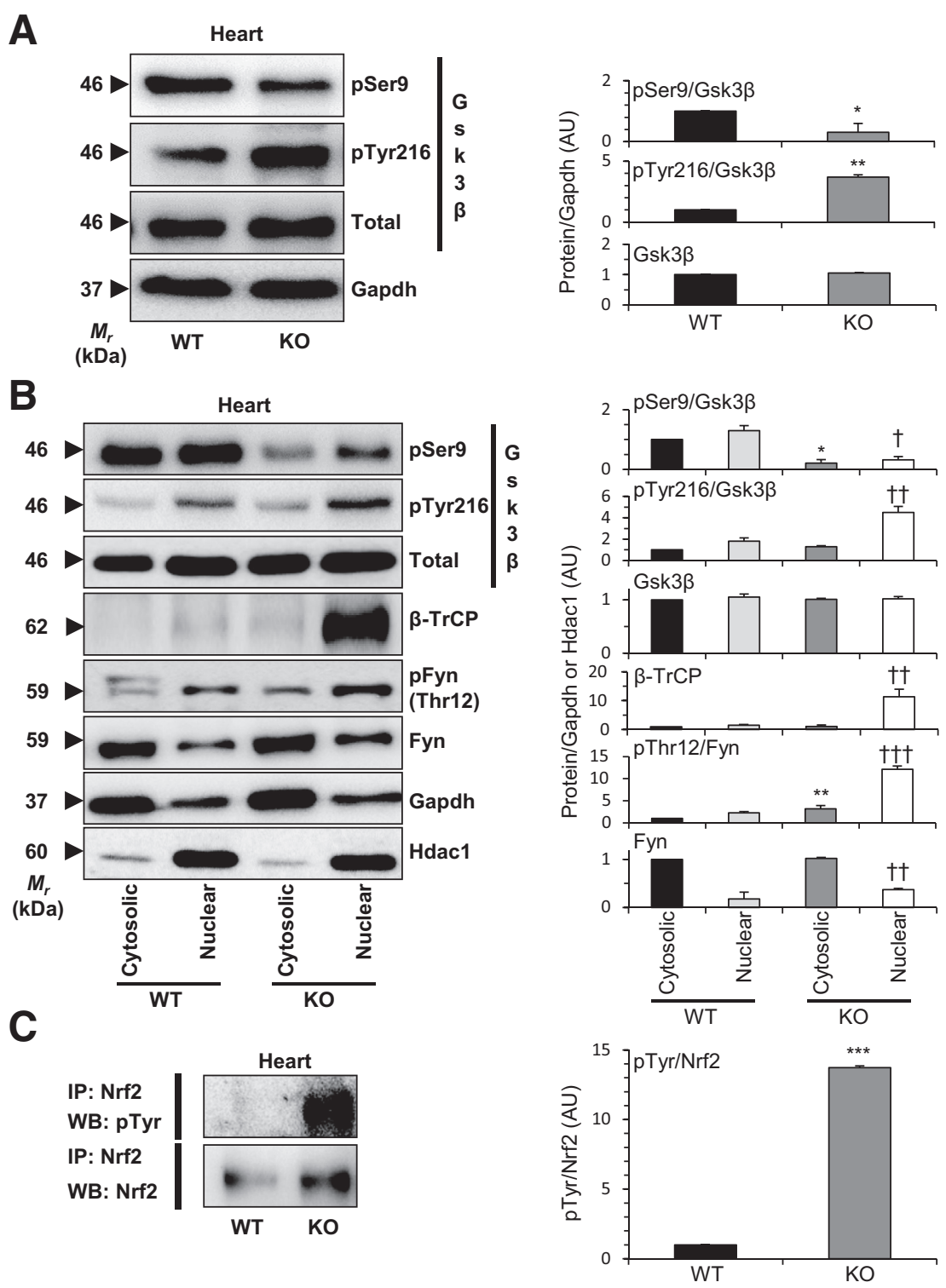

Figure 4 Western blot (WB) analysis demonstrating that the Gsk3 $\beta$-mediated nuclear Nrf2 export/degradation machinery is activated in the heart of MCK knockout (KO) relative to the wildtype (WT) mice. MCK frataxin K0 mice relative to WT mice were examined at 9 weeks of age. A: WB and densitometric analysis of phosphorylated (Ser9 and Tyr216) relative to total Gsk3 $\beta$ expression in total heart lysate. Glyceraldehyde-3phosphate dehydrogenase (Gapdh) was used as a loading control and implemented for normalization of protein loading. B: WB and densitometric analysis of the expression and subcellular localization of phosphorylated Gsk3 $\beta$ (Ser9 and Tyr216) relative to total Gsk3 $\beta$ expression, $\beta$-TrCP expression, and phosphorylated (pThr12) and total Fyn expression in the cytosolic and nuclear heart fractions. Gapdh and histone deacetylase-1 (Hdac1) were used as fractionation controls for cytosolic and nuclear fractions, respectively, and implemented for normalization of protein expression. C: Immunoprecipitation (IP) of Nrf2, followed by WB and densitometric analysis of phosphorylated Tyr (pTyr) levels of Nrf2 using an anti-pTyr antibody. WB analyses shown are typical of three to four experiments. Densitometry data are means \pm SEM $(\mathbf{A}-\mathbf{C}) . n=3$ to 4 experiments $(\mathbf{A}-\mathbf{C}) .{ }^{*} P<0.05,{ }^{* *} P<0.01$, and ${ }^{* * *} P<0.001$ versus WT or WT cytosolic; ${ }^{\dagger} P<0.05,{ }^{\dagger \dagger} P<0.01$, and ${ }^{{ }^{\dagger \dagger} P}<0.001$ versus WT nuclear. AU, arbitrary unit; $M_{\mathrm{r}}$, molecular mass.

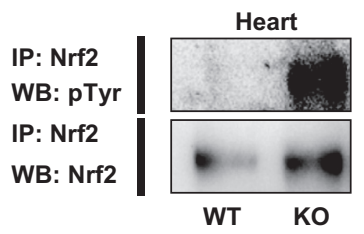

contrast, cellular expression of total Gsk3 $\beta$ was not significantly $(P>0.05)$ altered in the KO versus the WT littermates (Figure 4A). Collectively, these observations indicate that Gsk $3 \beta$ is activated (ie, phosphorylated at Tyr216) in the heart of KO mice relative to their WT littermates.

More important, because Gsk3 $\beta$-dependent mechanisms regulate Nrf2 expression in the nucleus, ${ }^{43-46}$ we then performed cellular fractionation studies of WT and KO heart lysates to examine the phosphorylation status and subcellular localization of Gsk3 $\beta$ (Figure 4B). As in Figure 3, C and $\mathrm{D}$, the expression levels of Gapdh and histone deacetylase-1 were examined as cytosolic and nuclear fraction markers, respectively. The $\mathrm{pSer} 9 / \mathrm{Gsk} 3 \beta$ ratio was significantly $(P<0.05)$ decreased in both the cytosolic and nuclear fractions (Figure 4B). On the other hand, the pTyr216/Gsk3 $\beta$ ratio was slightly $(P>0.05)$ increased in the cytosolic fraction in the KO versus WT mice, but significantly $(P<0.01)$ increased in the nuclear fraction in the $\mathrm{KO}$ mice relative to the WT mice (Figure 4B). In accordance with the results from the whole heart lysate (Figure 4A), total Gsk3 $\beta$ expression was not significantly $(P>0.05)$ altered in both the cytosolic and nuclear fractions (Figure 4B).

Activation of Gsk3 $\beta$ has been reported to directly phosphorylate Nrf2 at Ser338, ${ }^{45}$ which leads to recruitment of the $\beta$-TrCP. ${ }^{43,44}$ This protein is a substrate recognition subunit of the Skp1-Cul1-Rbx/Roc1 E3 ubiquitin ligase complex that targets Nrf2 phosphorylated at Ser338 for ubiquitination and subsequent nuclear export and/or degradation. $^{43-45}$ In the current studies, $\beta$-TrCP was observed to be predominantly localized in the nucleus of the $\mathrm{KO}$ heart, with a pronounced and significant $(P<0.01)$ increase in nuclear $\beta$-TrCP expression in the $\mathrm{KO}$ mice compared with their WT littermates (Figure 4B). Combined with the observed increased activation of Gsk3 $\beta$ in the $\mathrm{KO}$ mouse heart (Figure 4, A and B), these studies suggest increased 
Gsk3 $\beta$-mediated Nrf2 phosphorylation, leading to $\beta$-TrCP nuclear recruitment and subsequent Nrf2 nuclear export/ degradation. ${ }^{43-45}$

Another Gsk $3 \beta$-dependent mechanism of Nrf2 downregulation involves the controversial finding that phosphorylation of Nrf2 at Tyr568 leads to its nuclear efflux and degradation. ${ }^{46,48}$ This mechanism involves Gsk3 $\beta$ (Tyr216 phosphorylated form) activating the Src tyrosine kinase, Fyn, via threonine phosphorylation. ${ }^{46,49}$ This modification then results in the translocation of activated Fyn into the nucleus, ${ }^{46,49,50}$ where it has been suggested to phosphorylate the Nrf2 Tyr568 residue. ${ }^{46,48}$ Although the phosphorylation of Tyr568 is disputed, $^{48}$ the increased phosphorylation of Nrf2 tyrosine residues and Gsk3 $\beta$-Fyn activation have been linked to enhanced nuclear export by other investigators. ${ }^{46,49-51}$ To further examine and dissect the mechanism of the decreased nuclear Nrf2 levels in frataxin KO mice, the subcellular localization and phosphorylation of Fyn were assessed.

More important, phosphorylation of Fyn at Thr12 has been demonstrated to activate both its tyrosine kinase activity and its translocation into the nucleus, ${ }^{52,53}$ which may then lead to the phosphorylation Nrf2. ${ }^{46}$ Considering this, Fyn phosphorylation at $\mathrm{Thr} 12$ was examined (Figure 4B). As expected from the activation of Gsk3 $\beta$, the cytosolic and particularly the nuclear levels of the phosphorylated Fyn (Thr12)/Fyn ratio were significantly $(P<0.001$ to $P<0.01)$ elevated in the KO relative to WT littermate hearts (Figure 4B). Consistent with the nuclear accumulation of phosphorylated Fyn, ${ }^{46}$ nuclear expression of total Fyn was significantly $(P<0.01)$ greater in the $\mathrm{KO}$ mice relative to WT littermates (Figure 4B).

Considering the elevated Fyn phosphorylation in the KO heart, studies then examined the phosphorylation of Nrf2 tyrosine levels by probing immunoprecipitated Nrf2 using an anti-pTyr antibody (Figure 4C). This assessment of general tyrosine phosphorylation has been previously implemented by others to gauge Fyn kinase activity in this context. ${ }^{46,50}$ In addition, examination of general $\mathrm{Tyr}$ phosphorylation was also deemed appropriate, considering that there is no commercially available antibody against phosphorylated Tyr568 and the role of Tyr568 phosphorylation in Nrf2 regulation remains unclear. ${ }^{48}$ Our studies revealed a pronounced and significant $(P<0.001)$ increase of Nrf2 Tyr phosphorylation in the immunoprecipitate from KO hearts relative to the hearts from their WT littermates (Figure 4C). These data are consistent with the phosphorylation of Nrf2 Tyr residues by the Gsk3 $\beta$-Fyn axis in the KO mice, which could then be targeted for nuclear export. $^{46}$

Collectively, these data in Figure 4 demonstrate activation of the Gsk3 $\beta$-mediated nuclear export/degradation machinery of Nrf2 via the $\beta-\mathrm{TrCP}$ and/or Fyn mechanisms in the heart of $\mathrm{KO}$ mice relative to their WT littermates.
The Gsk3 $\beta$-Mediated Mechanism for Decreasing Nuclear Nrf2 Is Inactivated in the Skeletal Muscle of the KO Mice

As a relevant comparison to the heart (Figure 4), the levels of phosphorylated Gsk $3 \beta$ (inactivating pSer9 and activating pTyr216) were also examined in the skeletal muscle of MCK mice by Western blot analysis (Figure 5). In contrast to the total heart lysate (Figure $4 \mathrm{~A}$ ), the $\mathrm{pSer} 9 / \mathrm{Gsk} 3 \beta$ ratio in the total skeletal muscle lysate was slightly, but significantly $(P<0.05)$, increased in the KO relative to WT mouse, whereas the pTyr216/Gsk3 $\beta$ ratio was not significantly $(P>0.05)$ altered (Figure 5A). Similarly to the heart, total cellular Gsk3 $\beta$ expression in skeletal muscle was not significantly $(P>0.05)$ altered in $\mathrm{KO}$ mice relative to their WT littermates (Figure 5A). These observations suggest a tissue-specific alteration between the heart and skeletal muscle of KO MCK mice (namely, a decrease of the inactivating Ser9 phosphorylation of Gsk3 $\beta$ in the heart), whereas there was increased Ser9 phosphorylation in the skeletal muscle, indicating Gsk3 $\beta$ inactivation.

To enable a comparison with the heart (Figure 4B), the subcellular localization and/or phosphorylation of Gsk3 $\beta$, $\beta$ - TrCP, and Fyn was examined by performing cellular fractionation studies in WT and KO skeletal muscle lysates (Figure 5B). As in the studies above examining the heart, the cytosolic and nuclear fraction markers, Gapdh and histone deacetylase-1, respectively, were assessed as relative fraction controls. In the skeletal muscle, the inactivating pSer9/ Gsk $3 \beta$ ratio was significantly $(P<0.01)$ increased in the nuclear fraction of the $\mathrm{KO}$ mouse relative to the WT (Figure 5B). In contrast, the activating pTyr216/Gsk3 $\beta$ ratio demonstrated no significant $(P>0.05)$ change in both the nuclear and cytosolic fractions in the $\mathrm{KO}$ mice relative to their WT littermates (Figure 5B). The total Gsk $3 \beta$ expression was not significantly $(P>0.05)$ altered in both the cytosolic and nuclear fractions in the skeletal muscle (Figure 5B).

Examination of $\beta$-TrCP levels in the skeletal muscle demonstrated that, in $\mathrm{KO}$ mice, there was a significant $(P<0.01)$ decrease in the expression of both the cytosolic and nuclear $\beta$-TrCP relative to their respective levels in WT littermates (Figure 5B). This finding was in marked contrast to the increased $\beta$-TrCP levels in the heart nuclear fraction of the KO mice relative to the WT mice (Figure 4B), and may be caused by the increased inhibition of Gsk $3 \beta$ in the KO skeletal muscle (Figure 5, A and B).

Furthermore, in terms of Fyn phosphorylation status at Thr12, in contrast to the heart (Figure 4B), there was no significant $(P>0.05)$ alteration in the nuclear and cytosolic fractions of KO skeletal muscle relative to the WT (Figure 5B). Studies then examined the phosphorylation of Nrf2 by immunoprecipitation (as in Figure 4C), and this demonstrated no significant $(P>0.05)$ change of Nrf2 Tyr phosphorylation from KO relative to WT skeletal muscle (Figure 5C). These data are in contrast to the results assessing the heart in Figure $4 \mathrm{C}$, indicating that the 
A

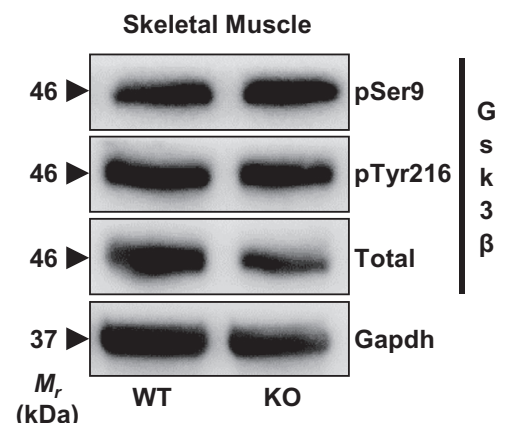

B

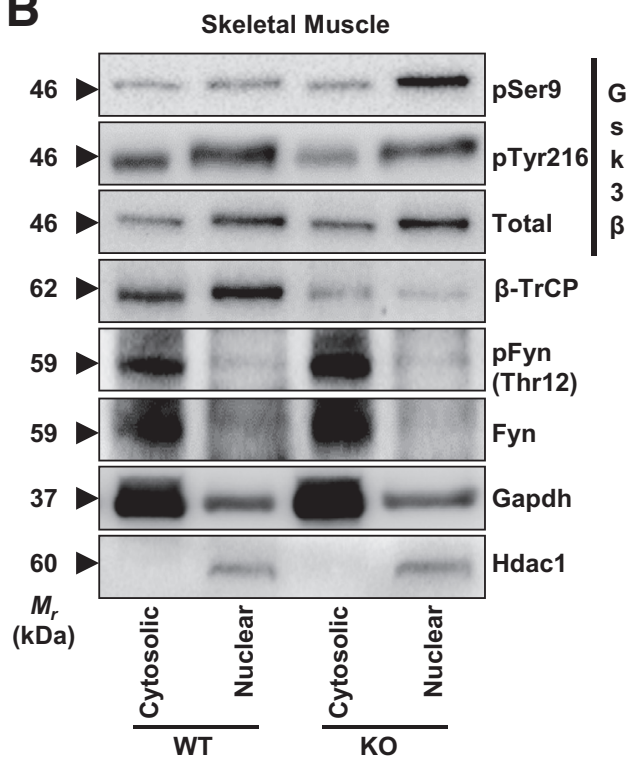

C

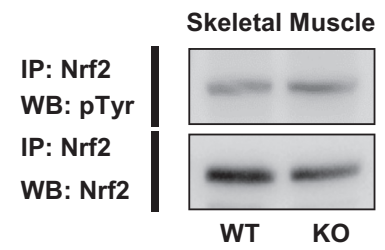

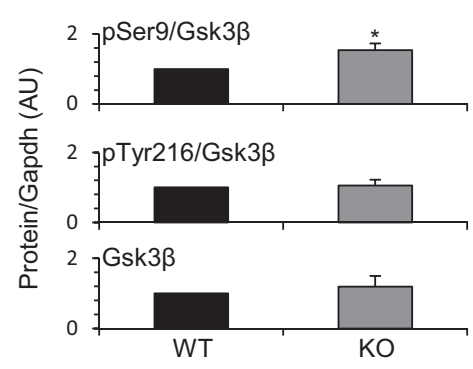
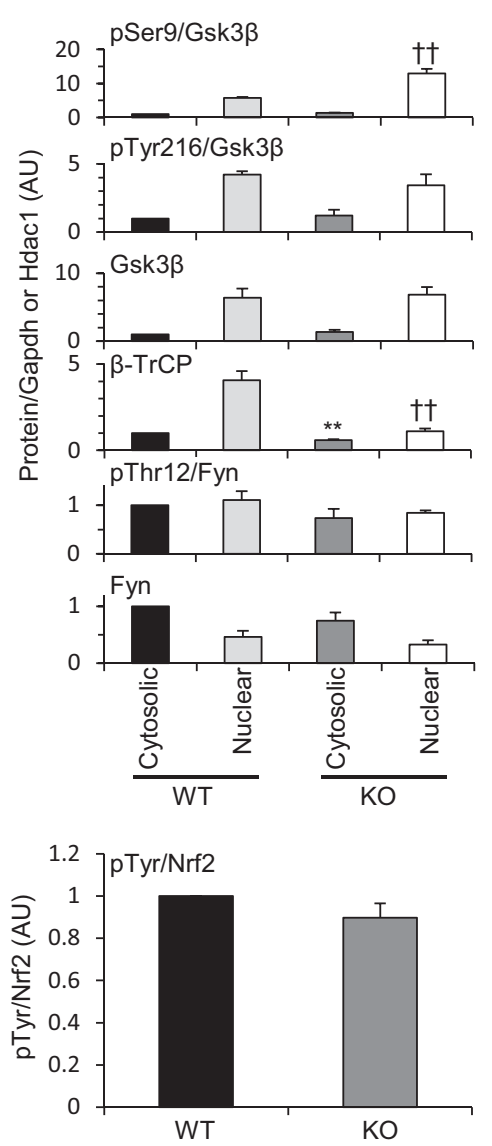

Figure 5 Western blot (WB) analysis demonstrating that the Gsk3ß-mediated nuclear Nrf2 export/degradation machinery is inactivated in the skeletal muscle of MCK knockout (KO) relative to the wild-type (WT) mice. MCK frataxin KO mice relative to WT mice were examined at 9 weeks of age. A: WB and densitometric analysis of phosphorylated (Ser9 and Tyr216) relative to total Gsk3 $\beta$ expression in total skeletal muscle lysate. Glyceraldehyde-3-phosphate dehydrogenase (Gapdh) was used as a loading control and implemented for normalization of protein loading. B: WB and densitometric analysis of phosphorylated Gsk3 $\beta$ (Ser9 and Tyr216) relative to total Gsk3 $\beta$ expression, $\beta$-TrCP expression, and phosphorylated (pThr12) and total Fyn expression in the cytosolic and nuclear skeletal muscle fractions. Gapdh and histone deacetylase-1 (Hdac1) were used as fractionation controls for cytosolic and nuclear fractions, respectively, and implemented for normalization of protein expression. C: Immunoprecipitation (IP) of Nrf2, followed by WB and densitometric analysis of phosphorylated Tyr (pTyr) levels of Nrf2 using an anti-pTyr antibody. WB analyses shown are typical of three to four experiments. Densitometry data are means \pm SEM $(\mathbf{A}-\mathbf{C}) . n=3$ to 4 experiments $(\mathbf{A}-\mathbf{C}) .{ }^{*} P<0.05$, ${ }^{* *} P<0.01$ versus WT or WT cytosolic; ${ }^{\dagger \dagger} P<0.01$ versus WT nuclear. AU, arbitrary unit; $M_{\mathrm{r}}$, molecular mass.
Gsk3ß-Fyn axis has not been activated in the skeletal muscle of the KO mice relative to the WT mice. Collectively, these results demonstrate an opposite and tissuespecific effect of frataxin deletion in the skeletal muscle compared with the heart of $\mathrm{KO}$ mice.

\section{Decreased Nrf2 Binding to the ARE Attenuates the} Antioxidant Response in $\mathrm{KO}$ Hearts

Considering the marked histopathology in the heart (Figure 1, A-F), the observed alterations in oxidative markers (Figure 2, A, C, E, G, and I), and the distinct reduction in $\mathrm{Nrf} 2$ expression in the total (Figure 3A) and nuclear (Figure 3C) fractions of the KO mouse heart, we then examined the ARE-binding activity in heart nuclear lysates using an electrophoretic mobility shift assay (Figure 6A). As demonstrated in Figure 6A, although there was a specific band representing protein binding to the ARE probe sequence in the WT nuclear sample, the binding was significantly $(P<0.01)$ reduced in the KO mice (Figure 6A). A 200-fold excess of unlabeled ARE nucleotide sequence acting as a specific competitor was able to significantly $(P<0.001$ to $P<0.01)$ reduce the detected protein-bound ARE probe in both WT and KO samples, demonstrating the specificity of the protein DNA-binding activity (Figure 6A). Hence, in agreement with the Western blot results indicating decreased $\mathrm{Nrf} 2$ in the nucleus of the KO heart (Figure 3C), these studies demonstrated that Nrf2 DNA-binding activity was markedly reduced in the MCK KO relative to WT littermates. This observation 

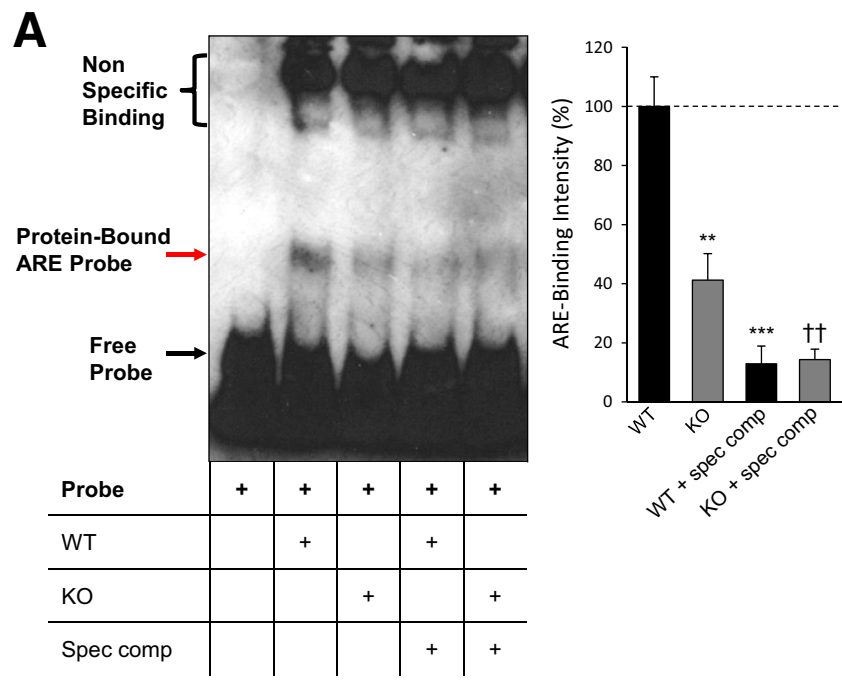

B

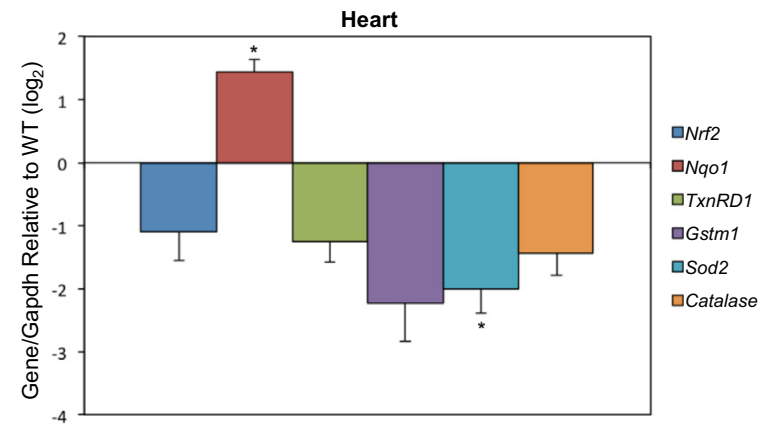

Figure 6 Electrophoretic mobility shift assay (EMSA) analysis demonstrates a decrease in antioxidant response element (ARE) binding and also a decrease in mRNA expression of Nrf2 and four of five target genes in the MCK knockout (KO) heart relative to the wild-type (WT) heart. Heart tissue from MCK frataxin KO mice relative to WT mice was examined at 9 weeks of age. A: Nuclear protein lysates from heart tissues of MCK WT and KO mice were prepared by cellular fractionation. The nuclear lysates $(20 \mu \mathrm{g})$ were then incubated with a 36 -bp biotin-labeled probe containing the mouse Nqo1 ARE sequence (5'-AGTCTAGAGTCACAGTGAGTGCCAAAATTTGAGCC-3', corresponding to nucleotides -451 to -416 ) in the presence or absence of the nonlabeled Nqo1 ARE sequence [ie, specific competitor (spec comp) probe]. EMSA analysis shown is typical of three experiments. Red and black arrows pointing to the gel indicate protein-bound ARE probe and free probe, respectively. Dashed line on densitometric analysis indicates $100 \%$. B: Quantification of the mRNA levels of ARE-containing genes (Nrf2, Nq01, TxnRD1, Gstm1, Sod2, and catalase) was examined by real-time quantitative RT-PCR and normalized to glyceraldehyde-3-phosphate dehydrogenase (Gapdh) mRNA expression. Densitometry data are means \pm SEM (A) or $\log _{2} \pm$ SEM (B). $n=3$ experiments (A and B); $n=5$ to 6 mice per genotype (B). ${ }^{*} P<0.05,{ }^{* *} P<0.01$, and ${ }^{* *} P<0.001$ versus $\mathrm{WT}$; ${ }^{\dagger} P<0.01$ versus $\mathrm{KO}$. AU, arbitrary unit.

suggested a potential reduction in Nrf2 antioxidant target gene mRNA levels in the KO heart.

To examine this latter possibility, we then assessed by real-time quantitative RT-PCR the expression of important cellular antioxidant gene targets of Nrf2 that contain an ARE within their promoter region (Figure 6B). These target genes included the following: $N r f 2, N A D(P) H$ dehydrogenase, Nqo1, TxnRD1, Gstm1, Sod2, and catalase. ${ }^{16,54-56}$ Despite the oxidative stress in the heart (Figure 2, A and I), and in agreement with the reduced total and nuclear Nrf2 protein levels (Figure 3, A and C) and Nrf2-ARE-binding activity (Figure 6A), we observed a significant $(P<0.05)$ decrease in Sod2 mRNA expression, together with a nonsignificant $(P>0.05)$ decrease in the levels of $N r f 2$, TxnRDI, Gstml, and catalase mRNA in the KO mice compared with their WT littermates (Figure 6B). Only Nqo1 mRNA levels were slightly, but significantly $(P<0.05)$, increased in the KO mice relative to WT littermates (Figure 6B). Notably, our previous microarray analysis examining MCK KO and WT mice (Gene Expression Omnibus data set GSE31208; https://www.ncbi.nlm.nih.gov/ $g e o)^{23}$ also identified a significant $(P<0.01)$ decrease in Sod 2 mRNA expression in the KO mice, whereas no other antioxidant genes were significantly altered. The decrease in Sod2 mRNA levels is in agreement with previous studies in mouse models of FA, ${ }^{13,57,58}$ and consistent with the finding that oxidative challenge is unable to induce Sod2 in cells from FA patients. ${ }^{13,59}$ Taken together, despite the marked pathology and increased oxidative products in the KO heart
(Figures 1, A-F, and 2, A, C, E, G, and I), the mRNA expression of $N r f 2$ and four of its five target genes were not upregulated in response to oxidative stress.

Intriguingly, despite the general decrease in mRNA levels, further examination of these downstream Nrf2 antioxidant targets at the protein level demonstrated contrasting results (Figure 7). In the heart, there was a significant $(P<0.001)$ increase in the expression of Nqo1, Gstm1, and TxnRD1 in the KO mice compared with their WT littermates, whereas the expression levels of Sod2 and catalase were unchanged (Figure 7, A and B). Notably, in yeast models of frataxin deficiency, Sod2 protein levels were also unchanged, despite high mitochondrial iron. ${ }^{60}$ In the skeletal muscle of KO mice, where no significant oxidative stress (Figure 2, B and J) or alteration in Nrf2 levels (Figure 3, B and D) was observed, there was a significant $(P<0.001$ to $P<0.01)$ increase in the protein levels of Nqo1, Gstm1, and catalase relative to WT littermates (Figure 7, A and C). In contrast, Sod2 and TxnRD1 protein expression remained unchanged (Figure 7, A and C). The reason for the increased expression of Nqo1, Gstm1, and catalase in the KO skeletal muscle was unclear.

\section{Discussion}

Our previous study demonstrated the marked functional and molecular alterations in the MCK KO heart relative to the WT heart, with the pathogenesis of the cardiomyopathy correlating with early and persistent eIF $2 \alpha$ phosphorylation, 

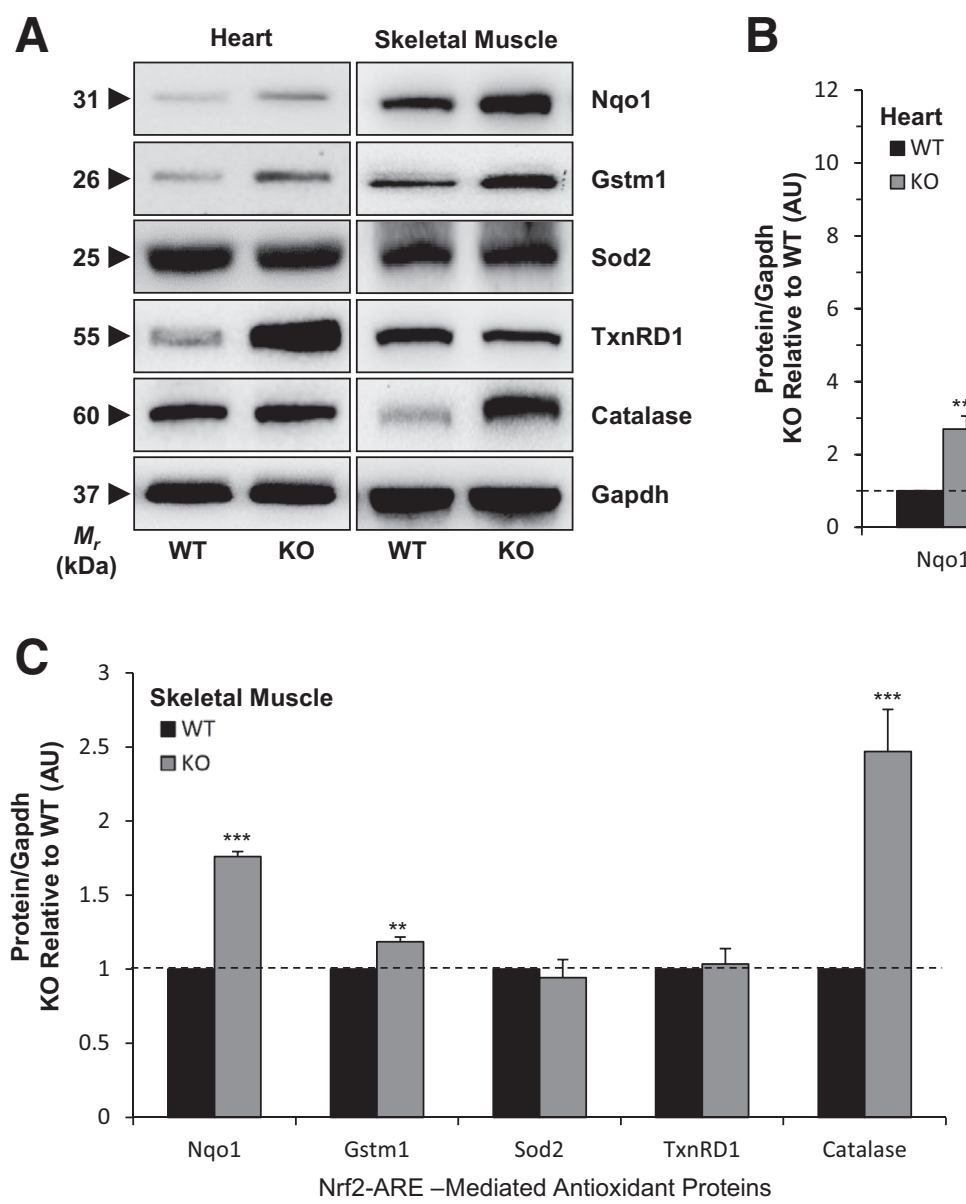

B

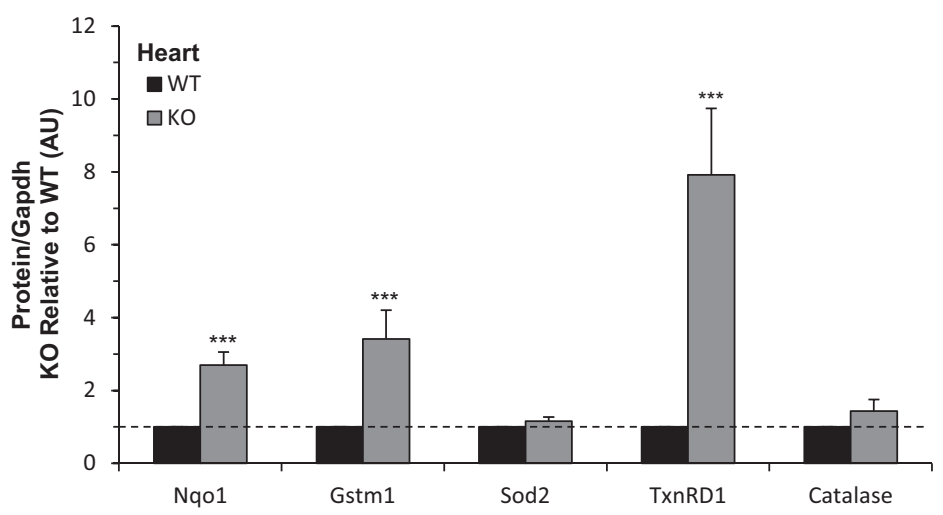

Figure 7 Western blot analysis demonstrates differential expression profile of antioxidant proteins in the heart and skeletal muscle of MCK wild-type (WT) and knockout (KO) mice. MCK frataxin KO mice relative to WT mice were examined at 9 weeks of age. Western blot (A) and densitometric (B and C) analyses of quinone 1 (Nqo1), glutatione-S-transferase Mu1 (Gstm1), superoxide dismutase 2 (Sod2), thioredoxin reductase 1 (TxnRD1), and catalase expression in total heart and total skeletal muscle lysate. Glyceraldehyde-3-phosphate dehydrogenase (Gapdh) was used as a loading control and implemented for normalization of protein loading. Dashed lines indicate the value for the WT littermates. Western blot analysis shown is typical of three to four experiments. Densitometry data are means \pm SEM (B and C). $n=3$ to 4 experiments (A-C). ${ }^{* *} P<0.01,{ }^{* * *} P<0.001$ versus WT. AU, arbitrary unit.

which precedes activation of autophagy and apoptosis. ${ }^{7}$ From our current investigation of Nrf2 signaling in the frataxin-deficient heart, we have demonstrated, for the first time, the mechanism responsible for the decrease of Nrf2 after loss of frataxin in the heart (Figure 8). This response in the heart appears paradoxical in the face of the observed oxidative stress that is evident from protein oxidation and GSH measurements (Figure 2, A, C, E, G, and I) and previous studies demonstrating the importance of Nrf2 in cardioprotection from oxidative damage in vivo. ${ }^{61,62}$

Moreover, a tissue-specific effect was observed; although frataxin deletion also occurred in the MCK skeletal muscle, no oxidative stress, histological abnormalities, or Nrf2 dysfunction was observed. These observations are in good agreement with the tissue-specific characteristics of FA, as reported by others in patients ${ }^{29,30}$ and in the MCK mouse model. ${ }^{26}$ In fact, previous studies have demonstrated that, in striking contrast to the heart, the skeletal muscle of the MCK model does not show any histological, ultrastructural, or biochemical defect, despite extensive Cre recombination. ${ }^{26}$ The differential between the heart and skeletal muscle may be attributable to the generally known fact that the heart relies on oxidative phosphorylation via the mitochondrion, with an almost exclusive dependence on aerobic metabolism. ${ }^{63}$ In contrast, the skeletal muscle is more dependent on cytosolic anaerobic glycolysis for its energy requirements. ${ }^{63}$ Thus, it can be suggested the mitochondrial dysfunction caused by frataxin deficiency ${ }^{64-66}$ has a greater impact on the metabolism of the heart relative to the skeletal muscle.

Despite oxidative stress in the MCK KO heart, our data demonstrate decreased total cellular and nuclear Nrf2 levels that correspond with increased Keap1 at 9 weeks of age (Figure 3, A and C). This observation suggests classic Keap1-mediated degradation of cytosolic Nrf $2,{ }^{18}$ which has not been previously characterized after frataxin deletion. Our studies, for the first time, demonstrate a significant increase in Keap1 in the frataxin-deficient heart that is well known to result in decreased cytosolic Nrf2 levels. ${ }^{18}$ Previous investigations using various models of frataxin deficiency have: i) not assessed Keap1 expression ${ }^{36}$; ii) indicated no alteration in Keap $1^{25}$; or iii) reported an alteration in cellular distribution of Keap1, but without increased expression. ${ }^{24}$ Moreover, the observed increase of Keap1 in 4-week-old $\mathrm{KO}$ heart, where there is no morphological or functional cardiac pathology, ${ }^{7}$ suggests the dysregulation of the Nrf2 pathway occurs relatively early and is not a consequence of the marked cardiomyopathy at 9 weeks of age.

The expression of Bach1, which is another key regulator of Nrf2 activity, was decreased in the frataxin-deficient 


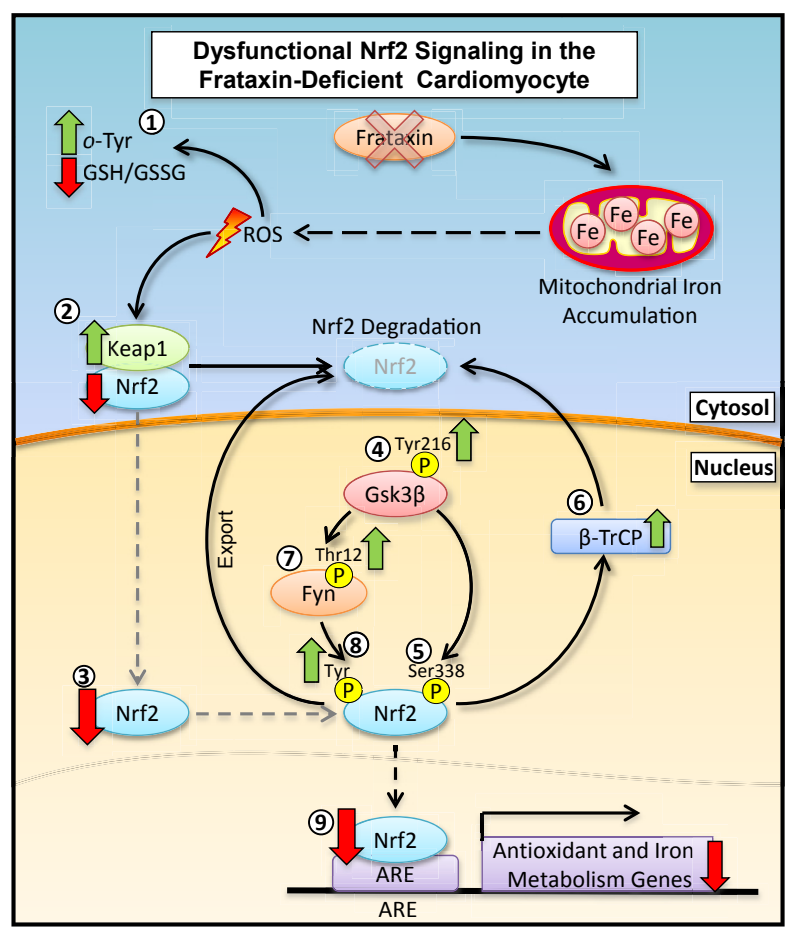

Figure 8 Schematic illustrating the mechanisms mediating the impaired Nrf2 response in the heart of MCK frataxin knockout (K0) mice. (1) Frataxin deficiency results in mitochondrial iron accumulation ${ }^{6,23,31}$ that could increase reactive oxygen species (ROS) generation and the production of oxidative products (Figure 2, A, C, E, G, and I). (2) Despite the presence of oxidative stress in the MCK KO heart, increased cytosolic Keap1 expression (Figure 3, A and C) could result in Keap1-mediated proteasomal degradation of Nrf2, ${ }^{18}$ decreasing its levels (Figure 3, A and C). (3) Reduced Nrf2 expression leads to decreased Nrf2 nuclear levels (Figure 3C). (4) Within the nucleus, increased Gsk3 $\beta$ activation (Tyr216 phosphorylation) (Figure 4B) may result in (5) direct Gsk3ß-mediated Nrf2 phosphorylation (Ser338 $8^{45}$ ) and (6) subsequent nuclear accumulation of $\beta-\operatorname{TrCP}$ (Figure $4 \mathrm{~B}$ ), which facilitates the decrease in nuclear Nrf2 (Figure $3 C$ ) via the known processes of nuclear export and/or degradation. ${ }^{18}$ (7) Alternatively, activated Gsk3 $\beta$ could increase phosphorylation of Fyn (Thr12) (Figure 4B) to enhance Fyn tyrosine kinase activity. ${ }^{52,53}$ (8) This, in turn, mediates Tyr phosphorylation of Nrf2 (Figure 4C), leading to a decrease in nuclear Nrf2 (Figure 3C), via its export. ${ }^{46}$ (9) These mechanisms culminate in decreased antioxidant response element (ARE)-binding activity (Figure $6 \mathrm{~A}$ ) and in a slight, but general, decrease in the expression of ARE-containing genes (Figure 6B) observed in the MCK frataxin KO heart. GSH, glutathione; GSSG, oxidized GSH.

heart. In the absence of heme, Bach1 acts as a repressor of Nrf2-DNA-binding activity ${ }^{22}$; as such, it is likely the decrease in nuclear Bach1 in the KO heart (Figure 3C) should facilitate access of Nrf2 to AREs. Nonetheless, the decreased Bach1 expression observed in the heart was surprising, because heme synthesis and heme levels are depressed in the heart of MCK KO mice, ${ }^{23}$ which should have led to increased nuclear Bach1 levels. ${ }^{22}$ Indeed, it is well known that on heme binding to Bach1, its DNAbinding activity is reduced and nuclear export is increased. ${ }^{22}$

Apart from the dysfunction in the classic Keap1/Bach1 system for controlling Nrf2 activity in the KO heart, we demonstrate, for the first time, activation of Nrf2 nuclear export/degradation machinery via Gsk3 $\beta$-mediated Nrf2 phosphorylation either directly or through Fyn kinase. ${ }^{43-46}$ In fact, in the KO heart, there were increased levels of the activating phosphorylation of Gsk3 $\beta$ (Tyr216) (Figure 4, A and B) and Fyn kinase (Thr12) (Figure 4B), as well as increased Tyr phosphorylation of Nrf2 (Figure 4C), that are known to result in Nrf2 nuclear export. ${ }^{46,50}$ This effect, in conjunction with the enhanced $\beta$-TrCP expression (Figure 4B), could facilitate the degradation of nuclear Nrf2 by acting as a substrate for the E3 proteasome complex of Cullin-1 and Rbx $1 .{ }^{43}$ Collectively, the alterations in the Nrf2 pathway by these mechanisms could be responsible for the decreased Nrf2 protein levels, reduced ARE binding, and the generally depressed expression of ARE-containing antioxidant defense genes in the frataxin-deficient heart.

Despite Nrf2 down-regulation in the heart (Figure 3, A and C) and a slight, but general, decrease in the mRNA levels of it downstream antioxidant targets (Figure 6B), the protein levels of some of these antioxidant targets were increased in the $\mathrm{KO}$ mice (Figure 7, A-C). A lack of correlation between mRNA and protein expression is well known in the literature and is attributable to the existence of post-transcriptional mechanisms. These can include RNAbinding proteins, such as iron regulatory protein- $1^{67,68}$ and others ${ }^{69}$ that can modulate mRNA stability and translation; and miRNAs, ${ }^{70}$ which silence mRNA translation and can lead to little correlation between mRNA levels and protein expression. In addition, there are also post-translational mechanisms that can rapidly degrade proteins (eg, proteasome and also the lysosome via autophagy ${ }^{71}$ ), which can also lead to a lack of correlation between mRNA and protein levels. Furthermore, these processes can be dysregulated during disease, ${ }^{71,72}$ leading to additional complexity in terms of understanding the balance between mRNA and protein expression.

Even with the marked down-regulation of Nrf2 in the heart, there were still appreciable mRNA levels of its target effector genes, with some of these being upregulated (ie, NQO1). This could be attributable to the activity of other transcription factors (eg, peroxisome proliferator-activated receptor $\gamma$ and forkhead box O) that actively target these critical Nrf2 downstream effectors ${ }^{73-75}$ and could potentially compensate for Nrf2 dysfunction. In fact, our previous studies examining the MCK heart demonstrated that the mRNA and protein expression of heme oxygenase 1, which is also an ARE-containing gene and Nrf2 target, ${ }^{44}$ was markedly and significantly increased, ${ }^{23}$ despite the marked depression of Nrf2 activity demonstrated herein. Together, these observations indicate that other mechanisms can at least partially compensate for the depression in Nrf2 levels. Nonetheless, despite the compensation observed in terms of antioxidant response, an overall state of oxidative stress was evident in the KO heart, as demonstrated by increased levels of the phenylalanine oxidation product, $o$-Tyr, and the depressed GSH/GSSG ratio (Figure 2).

Collectively, the current study provides a rationale for antioxidant supplementation to enhance cardiac GSH 
(thereby increasing the GSH/GSSG ratio) through administration of antioxidants, such as $\mathrm{N}$-acetylcysteine (NAC) ${ }^{76-78}$ Intriguingly, NAC has been demonstrated to increase Nrf2 expression in vivo, ${ }^{76,79}$ which should bolster antioxidant defense. This is important because the Nrf2 response is decreased in the heart, which leads to oxidative stress (Figure 2), probably because of a less than adequate response of the battery of antioxidant response proteins downstream of Nrf2. Enhancing the upregulation of these proteins (eg, catalase and SOD, which did not display any significant increase in levels) (Figure 7, A-C) could be important to target. In addition, NAC is a Federal Drug Administration-approved $\operatorname{drug}^{77}$ and has been shown to have protective effects against cytotoxicity in nondifferentiated frataxin-deficient cell types, because of its ability to prevent ROS-induced cytotoxicity. ${ }^{78}$ This is significant, because previous studies in yeast mutants lacking frataxin have also shown that NAC supplements cellular GSH and prevents iron-induced toxicity, with cell survival increasing by two to four orders of magnitude. ${ }^{80}$

Considering the potential role of iron in the oxidative stress and pathogenesis of FA, and the beneficial effects of NAC in cellular systems of this disease, a potential therapeutic modality could include the combination of NAC with chelators (eg, pyridoxal isonicotinoyl hydrazone) that have been demonstrated to mobilize mitochondrial iron accumulation and inhibit oxidative stress. ${ }^{31,81,82}$ This could lead to a rationalized treatment for FA in the absence of a therapy to replace frataxin function. This is particularly relevant, because of the following: i) NAC can rescue low GSH levels, ${ }^{83}$ which were demonstrated to be low in the heart of the MCK mouse (Figure 2I) and prevent deleterious ROS-induced tissue damage; ii) in addition to its ability to directly react with ROS to prevent their deleterious activity, NAC also restitutes GSH ${ }^{84}$ which could be crucial because GSH plays a role in iron sulfur cluster assembly, ${ }^{85,86}$ a key defect in $\mathrm{FA}^{66}$; and iii) NAC has been shown to increase Nrf2 expression. ${ }^{76,79}$ Together, by removing an important oxidative insult and supplementing GSH, NAC could ameliorate ROS generation, bolster antioxidant defense by increasing Nrf2 expression, and aid the synthesis of critical iron sulfur clusters.

Although there is clear evidence demonstrating that frataxin deficiency results in increased ROS generation,,${ }^{9,10}$ the reason why a loss of frataxin leads to a decrease in Nrf2 expression remains uncertain. One possible mechanism could be related to the recent finding that intracellular iron levels can be increased through $\mathrm{Nrf} 2$ degradation and decreased Nrf2-induced expression of the iron export protein, ferroportin $1 .^{87}$ This is relevant, because ferroportin 1 is involved in iron release from cells, and we previously demonstrated the frataxin KO heart is in a cytosolic irondeficient state, where ferroportin 1 expression is decreased relative to WT mice. ${ }^{23}$ Such a decrease in ferroportin1 expression could reduce iron export and, thus, aid in preventing the iron deficit. ${ }^{23}$ Hence, the inhibition of Nrf2 expression observed herein may be part of an attempt by cellular regulatory mechanisms to decrease ferroportin 1 expression to reduce cellular iron efflux and, thus, restore intracellular iron levels in the absence of frataxin.

In summary, our study demonstrates, for the first time in the heart, that frataxin deficiency results in significant alterations in the cellular redox homeostasis mediated by Nrf2. Furthermore, this is the first investigation to dissect the mechanism of how loss of frataxin in the heart results in Nrf2 deficiency (namely, through increased cytosolic Keap1 levels and activation of nuclear Nrf2 export/degradation machinery via Gsk3 $\beta$ signaling). These effects lead to a general decrease in Nrf2 binding to the ARE of target genes involved in antioxidant defense. Hence, despite evidence of marked redox stress in the frataxin-deficient heart, the major antioxidant defense mechanism mediated via $\mathrm{Nrf2}$ is dysfunctional, and this could play a role in the cardiac pathology observed in FA.

\section{Acknowledgments}

We thank Drs. Danuta Kalinowski, Zaklina Kovacevic, Angelica Merlot, Sumit Sahni, Patric Jansson, Darius Lane, and Hiu Chuen Lok (Bosch Institute, University of Sydney) for careful assessment of the manuscript before submission.

\section{Supplemental Data}

Supplemental material for this article can be found at doi:https://doi.org/10.1016/j.ajpath.2017.08.021.

\section{References}

1. Campuzano V, Montermini L, Molto MD, Pianese L, Cossee M, Cavalcanti F, Monros E, Rodius F, Duclos F, Monticelli A, Zara F, Canizares J, Koutnikova H, Bidichandani SI, Gellera C, Brice A, Trouillas P, De Michele G, Filla A, De Frutos R, Palau F, Patel PI, Di Donato S, Mandel JL, Cocozza S, Koenig M, Pandolfo M: Friedreich's ataxia: autosomal recessive disease caused by an intronic GAA triplet repeat expansion. Science 1996, 271:1423-1427

2. Campuzano V, Montermini L, Lutz Y, Cova L, Hindelang C, Jiralerspong S, Trottier Y, Kish SJ, Faucheux B, Trouillas P, Authier FJ, Durr A, Mandel JL, Vescovi A, Pandolfo M, Koenig M: Frataxin is reduced in Friedreich ataxia patients and is associated with mitochondrial membranes. Hum Mol Genet 1997, 6:1771-1780

3. Koeppen AH: Friedreich's ataxia: pathology, pathogenesis, and molecular genetics. J Neurol Sci 2011, 303:1-12

4. Payne RM, Pride PM, Babbey CM: Cardiomyopathy of Friedreich's ataxia: use of mouse models to understand human disease and guide therapeutic development. Pediatr Cardiol 2011, 32:366-378

5. Michael S, Petrocine SV, Qian J, Lamarche JB, Knutson MD, Garrick MD, Koeppen AH: Iron and iron-responsive proteins in the cardiomyopathy of Friedreich's ataxia. Cerebellum 2006, 5:257-267

6. Whitnall M, Rahmanto YS, Huang MLH, Saletta F, Lok HC, Gutierrez L, Lazaro FJ, Fleming AJ, St Pierre TG, Mikhael MR, Ponka P, Richardson DR: Identification of nonferritin mitochondrial iron deposits in a mouse model of Friedreich ataxia. Proc Natl Acad Sci U S A 2012, 109:20590-20595

7. Huang ML, Sivagurunathan S, Ting S, Jansson PJ, Austin CJ, Kelly M, Semsarian C, Zhang D, Richardson DR: Molecular and 
functional alterations in a mouse cardiac model of Friedreich ataxia: activation of the integrated stress response, eIF2alpha phosphorylation, and the induction of downstream targets. Am J Pathol 2013, 183: $745-757$

8. Dröge W: Free radicals in the physiological control of cell function. Physiol Rev 2002, 82:47-95

9. Schulz J, Dehmer T, Schöls L, Mende H, Hardt C, Vorgerd M, Bürk K, Matson W, Dichgans J, Beal M, Bogdanov M: Oxidative stress in patients with Friedreich ataxia. Neurology 2000, 55: $1719-1721$

10. Sparaco M, Gaeta LM, Santorelli FM, Passarelli C, Tozzi G, Bertini E, Simonati A, Scaravilli F, Taroni F, Duyckaerts C, Feleppa M, Piemonte F: Friedreich's ataxia: oxidative stress and cytoskeletal abnormalities. J Neurol Sci 2009, 287:111-118

11. Emond M, Lepage G, Vanasse M, Pandolfo M: Increased levels of plasma malondialdehyde in Friedreich ataxia. Neurology 2000, 55: $1752-1753$

12. Pastore A, Tozzi G, Gaeta LM, Bertini E, Serafini V, Cesare SD, Bonetto V, Casoni F, Carrozzo R, Federici G, Piemonte F: Actin glutathionylation increases in fibroblasts of patients with Friedreich's ataxia: a potential role in the pathogenesis of the disease. J Biol Chem 2003, 278:42588-42595

13. Chantrel-Groussard K, Geromel V, Puccio H, Koenig M, Munnich A, Rotig A, Rustin P: Disabled early recruitment of antioxidant defenses in Friedreich's ataxia. Hum Mol Genet 2001, 10:2061-2067

14. Kensler TW, Wakabayashi N, Biswal S: Cell survival responses to environmental stresses via the Keap1-Nrf2-ARE pathway. Annu Rev Pharmacol Toxicol 2007, 47:89-116

15. Nguyen T, Nioi P, Pickett CB: The Nrf2-antioxidant response element signaling pathway and its activation by oxidative stress. J Biol Chem 2009, 284:13291-13295

16. Nioi P, McMahon M, Itoh K, Yamamoto M, Hayes JD: Identification of a novel Nrf2-regulated antioxidant response element (ARE) in the mouse $\mathrm{NAD}(\mathrm{P}) \mathrm{H}$ :quinone oxidoreductase 1 gene: reassessment of the ARE consensus sequence. Biochem J 2003, 374:337-348

17. Niture SK, Khatri R, Jaiswal AK: Regulation of Nrf2: an update. Free Radic Biol Med 2014, 66:36-44

18. Bryan HK, Olayanju A, Goldring CE, Park BK: The Nrf2 cell defence pathway: Keap1-dependent and -independent mechanisms of regulation. Biochem Pharmacol 2013, 85:705-717

19. Dhakshinamoorthy S, Jain AK, Bloom DA, Jaiswal AK: Bach1 competes with Nrf2 leading to negative regulation of the antioxidant response element (ARE)-mediated $\mathrm{NAD}(\mathrm{P}) \mathrm{H}$ : quinone oxidoreductase 1 gene expression and induction in response to antioxidants. $\mathrm{J}$ Biol Chem 2005, 280:16891-16900

20. Kaspar JW, Jaiswal AK: Antioxidant-induced phosphorylation of tyrosine 486 leads to rapid nuclear export of Bach1 that allows Nrf2 to bind to the antioxidant response element and activate defensive gene expression. J Biol Chem 2010, 285:153-162

21. Sun J, Brand M, Zenke Y, Tashiro S, Groudine M, Igarashi K: Heme regulates the dynamic exchange of Bach1 and NF-E2-related factors in the Maf transcription factor network. Proc Natl Acad Sci U S A 2004, 101:1461-1466

22. Suzuki H, Tashiro S, Hira S, Sun J, Yamazaki C, Zenke Y, IkedaSaito M, Yoshida M, Igarashi K: Heme regulates gene expression by triggering Crm1-dependent nuclear export of Bach1. EMBO J 2004, 23:2544-2553

23. Huang ML-H, Becker EM, Whitnall M, Rahmanto YS, Ponka P, Richardson DR: Elucidation of the mechanism of mitochondrial iron loading in Friedreich's ataxia by analysis of a mouse mutant. Proc Natl Acad Sci U S A 2009, 106:16381-16386

24. Paupe V, Dassa EP, Goncalves S, Auchère F, Lönn M, Holmgren A, Rustin P: Impaired nuclear Nrf2 translocation undermines the oxidative stress response in Friedreich ataxia. PLoS One 2009, 4: e4253

25. Shan Y, Schoenfeld RA, Hayashi G, Napoli E, Akiyama T, Iodi Carstens M, Carstens EE, Pook MA, Cortopassi GA: Frataxin deficiency leads to defects in expression of antioxidants and Nrf2 expression in dorsal root ganglia of the Friedreich's ataxia YG8R mouse model. Antioxid Redox Signal 2013, 19:1481-1493

26. Puccio H, Simon D, Cossee M, Criqui-Filipe P, Tiziano F, Melki J, Hindelang C, Matyas R, Rustin P, Koenig M: Mouse models for Friedreich ataxia exhibit cardiomyopathy, sensory nerve defect and Fe-S enzyme deficiency followed by intramitochondrial iron deposits. Nat Genet 2001, 27:181-186

27. Schneider CA, Rasband WS, Eliceiri KW: NIH Image to ImageJ: 25 years of image analysis. Nat Methods 2012, 9:671-675

28. Hawkins CL, Morgan PE, Davies MJ: Quantification of protein modification by oxidants. Free Radic Biol Med 2009, 46:965-988

29. Harding AE: Friedreich's ataxia: a clinical and genetic study of 90 families with an analysis of early diagnostic criteria and intrafamilial clustering of clinical features. Brain 1981, 104:589-620

30. Rotig A, de Lonlay P, Chretien D, Foury F, Koenig M, Sidi D, Munnich A, Rustin P: Aconitase and mitochondrial iron-sulphur protein deficiency in Friedreich ataxia. Nat Genet 1997, 17:215-217

31. Whitnall M, Rahmanto YS, Sutak R, Xu X, Becker EM, Mikhael MR, Ponka P, Richardson DR: The MCK mouse heart model of Friedreich's ataxia: alterations in iron-regulated proteins and cardiac hypertrophy are limited by iron chelation. Proc Natl Acad Sci U S A 2008, 105:9757-9762

32. Owen J, Butterfield DA: Measurement of oxidized/reduced glutathione ratio. Edited by Bross P, Gregersen N. In Protein Misfolding and Cellular Stress in Disease and Aging. New York, NY: Springer Science+Business Media, 2010. pp. 269-277

33. Davies MJ, Fu S, Wang H, Dean RT: Stable markers of oxidant damage to proteins and their application in the study of human disease. Free Radic Biol Med 1999, 27:1151-1163

34. Huang Q, Aluise CD, Joshi G, Sultana R, St Clair DK, Markesbery WR, Butterfield DA: Potential in vivo amelioration by Nacetyl-L-cysteine of oxidative stress in brain in human double mutant APP/PS-1 knock-in mice: toward therapeutic modulation of mild cognitive impairment. J Neurosci Res 2010, 88:2618-2629

35. Anderson ME: Glutathione: an overview of biosynthesis and modulation. Chem Biol Interact 1998, 111-112:1-14

36. D’Oria V, Petrini S, Travaglini L, Priori C, Piermarini E, Petrillo S, Carletti B, Bertini E, Piemonte F: Frataxin deficiency leads to reduced expression and impaired translocation of NF-E2-related factor (Nrf2) in cultured motor neurons. Int J Mol Sci 2013, 14: 7853-7865

37. Jyrkkänen $\mathrm{H}-\mathrm{K}$, Kuosmanen $\mathrm{S}$, Heinäniemi $\mathrm{M}$, Laitinen $\mathrm{H}$, Kansanen E, Mella-Aho E, Leinonen H, Ylä-Herttuala S, Levonen AL: Novel insights into the regulation of antioxidant-response-element mediated gene expression by electrophiles: induction of the transcriptional repressor BACH1 by Nrf2. Biochem J 2011, 440: $167-174$

38. Lee O-H, Jain AK, Papusha V, Jaiswal AK: An auto-regulatory loop between stress sensors INrf2 and Nrf2 controls their cellular abundance. J Biol Chem 2007, 282:36412-36420

39. Itoh K, Wakabayashi N, Katoh Y, Ishii T, Igarashi K, Engel JD, Yamamoto M: Keap1 represses nuclear activation of antioxidant responsive elements by Nrf2 through binding to the amino-terminal Neh2 domain. Genes Dev 1999, 13:76-86

40. Roy Chowdhury S, Sengupta S, Biswas S, Sinha TK, Sen R, Basak RK, Adhikari B, Bhattacharyya A: Bacterial fucose-rich polysaccharide stabilizes MAPK-mediated Nrf2/Keap1 signaling by directly scavenging reactive oxygen species during hydrogen peroxide-induced apoptosis of human lung fibroblast cells. PLoS One 2014, 9:e113663

41. Velichkova M, Hasson T: Keap1 regulates the oxidation-sensitive shuttling of Nrf2 into and out of the nucleus via a Crm1-dependent nuclear export mechanism. Mol Cell Biol 2005, 25:4501-4513

42. Coppola G, Marmolino D, Lu D, Wang Q, Cnop M, Rai M, Acquaviva F, Cocozza S, Pandolfo M, Geschwind DH: Functional genomic analysis of frataxin deficiency reveals tissue-specific 
alterations and identifies the PPARgamma pathway as a therapeutic target in Friedreich's ataxia. Hum Mol Genet 2009, 18:2452-2461

43. Rada P, Rojo AI, Chowdhry S, McMahon M, Hayes JD, Cuadrado A: $\mathrm{SCF} / \beta-\mathrm{TrCP}$ promotes glycogen synthase kinase 3-dependent degradation of the Nrf2 transcription factor in a Keap1-independent manner. Mol Cell Biol 2011, 31:1121-1133

44. Rada P, Rojo AI, Evrard-Todeschi N, Innamorato NG, Cotte A, Jaworski T, Tobón-Velasco JC, Devijver H, García-Mayoral MF, Van Leuven F, Hayes JD, Bertho G, Cuadrado A: Structural and functional characterization of Nrf2 degradation by the glycogen synthase kinase 3/ß-TrCP axis. Mol Cell Biol 2012, 32: 3486-3499

45. Chowdhry S, Zhang Y, McMahon M, Sutherland C, Cuadrado A, Hayes JD: Nrf2 is controlled by two distinct beta-TrCP recognition motifs in its Neh6 domain, one of which can be modulated by GSK-3 activity. Oncogene 2013, 32:3765-3781

46. Jain AK, Jaiswal AK: GSK-3ß acts upstream of Fyn kinase in regulation of nuclear export and degradation of NF-E2 related factor 2. J Biol Chem 2007, 282:16502-16510

47. Grimes CA, Jope RS: The multifaceted roles of glycogen synthase kinase $3 \beta$ in cellular signaling. Prog Neurobiol 2001, 65:391-426

48. Jain AK, Jaiswal AK: Phosphorylation of tyrosine 568 controls nuclear export of Nrf2. J Biol Chem 2006, 281:12132-12142

49. Shang G, Tang X, Gao P, Guo F, Liu H, Zhao Z, Chen Q, Jiang T, Zhang N, Li H: Sulforaphane attenuation of experimental diabetic nephropathy involves GSK-3 beta/Fyn/Nrf2 signaling pathway. J Nutr Biochem 2015, 26:596-606

50. Mobasher MA, Gonzalez-Rodriguez A, Santamaria B, Ramos S, Martin MA, Goya L, Rada P, Letzig L, James LP, Cuadrado A, Martin-Perez J, Simpson KJ, Muntane J, Valverde AM: Protein tyrosine phosphatase 1B modulates GSK3beta/Nrf2 and IGFIR signaling pathways in acetaminophen-induced hepatotoxicity. Cell Death Dis 2013, 4:e626

51. Xue M, Momiji H, Rabbani N, Barker G, Bretschneider T, Shmygol A, Rand DA, Thornalley PJ: Frequency modulated translocational oscillations of Nrf2 mediate the antioxidant response element cytoprotective transcriptional response. Antioxid Redox Signal 2015, 23:613-629

52. He Z, Tang F, Ermakova S, Li M, Zhao Q, Cho Y-Y, Ma W-Y, Choi H-S, Bode AM, Yang CS, Dong Z: Fyn is a novel target of (-)-epigallocatechin gallate in the inhibition of JB6 Cl41 cell transformation. Mol Carcinog 2008, 47:172-183

53. He Z, Cho Y-Y, Ma W-Y, Choi HS, Bode AM, Dong Z: Regulation of ultraviolet B-induced phosphorylation of histone $\mathrm{H} 3$ at serine 10 by Fyn kinase. J Biol Chem 2005, 280:2446-2454

54. Hintze KJ, Wald KA, Zeng H, Jeffery EH, Finley JW: Thioredoxin reductase in human hepatoma cells is transcriptionally regulated by sulforaphane and other electrophiles via an antioxidant response element. J Nutr 2003, 133:2721-2727

55. Chanas SA, Jiang Q, McMahon M, McWalter GK, McLellan LI, Elcombe CR, Henderson CJ, Wolf CR, Moffat GJ, Itoh K, Yamamoto M, Hayes JD: Loss of the Nrf2 transcription factor causes a marked reduction in constitutive and inducible expression of the glutathione S-transferase Gsta1, Gsta2, Gstm1, Gstm2, Gstm3 and Gstm4 genes in the livers of male and female mice. Biochem J 2002, 365:405-416

56. Reisman SA, Yeager RL, Yamamoto M, Klaassen CD: Increased Nrf2 activation in livers from Keap1-knockdown mice increases expression of cytoprotective genes that detoxify electrophiles more than those that detoxify reactive oxygen species. Toxicol Sci 2009 , 108:35-47

57. Seznec H, Simon D, Bouton C, Reutenauer L, Hertzog A, Golik P, Procaccio V, Patel M, Drapier J-C, Koenig M, Puccio H: Friedreich ataxia: the oxidative stress paradox. Hum Mol Genet 2005, 14: 463-474

58. Sandi C, Sandi M, Jassal H, Ezzatizadeh V, Anjomani-Virmouni S, Al-Mahdawi S, Pook MA: Generation and characterisation of
Friedreich ataxia YG8R mouse fibroblast and neural stem cell models. PLoS One 2014, 9:e89488

59. Jiralerspong S, Ge B, Hudson TJ, Pandolfo M: Manganese superoxide dismutase induction by iron is impaired in Friedreich ataxia cells. FEBS Lett 2001, 509:101-105

60. Yang M, Cobine PA, Molik S, Naranuntarat A, Lill R, Winge DR, Culotta VC: The effects of mitochondrial iron homeostasis on cofactor specificity of superoxide dismutase 2. EMBO J 2006, 25: $1775-1783$

61. Li J, Ichikawa T, Villacorta L, Janicki JS, Brower GL, Yamamoto M, Cui T: Nrf2 protects against maladaptive cardiac responses to hemodynamic stress. Arterioscler Thromb Vasc Biol 2009, 29: $1843-1850$

62. Wang W, Li S, Wang H, Li B, Shao L, Lai Y, Horvath G, Wang Q, Yamamoto M, Janicki JS, Wang XL, Tang D, Cui T: Nrf2 enhances myocardial clearance of toxic ubiquitinated proteins. J Mol Cell Cardiol 2014, 72:305-315

63. Berg JM, Tymoczko JL, Stryer L: Biochemistry. ed 5. New York City: W H Freeman, 2002

64. Huang ML-H, Lane DJR, Richardson DR: Mitochondrial mayhem: the mitochondrion as a modulator of iron metabolism and its role in disease. Antioxid Redox Signal 2011, 15:3003-3019

65. Richardson DR, Lane DJR, Becker EM, Huang MLH, Whitnall M, Rahmanto YS, Sheftel AD, Ponka P: Mitochondrial iron trafficking and the integration of iron metabolism between the mitochondrion and cytosol. Proc Natl Acad Sci U S A 2010, 107:10775-10782

66. Vaubel RA, Isaya G: Iron-sulfur cluster synthesis, iron homeostasis and oxidative stress in Friedreich ataxia. Mol Cell Neurosci 2013, 55: $50-61$

67. Hentze MW, Kuhn LC: Molecular control of vertebrate iron metabolism: mRNA-based regulatory circuits operated by iron, nitric oxide, and oxidative stress. Proc Natl Acad Sci U S A 1996, 93: $8175-8182$

68. Richardson DR, Ponka P: The molecular mechanisms of the metabolism and transport of iron in normal and neoplastic cells. Biochim Biophys Acta 1997, 1331:1-40

69. Aguilera G, Volpi S, Rabadan-Diehl C: Transcriptional and posttranscriptional mechanisms regulating the rat pituitary vasopressin V1b receptor gene. J Mol Endocrinol 2003, 30:99-108

70. Tabernero A, Gangoso E, Jaraiz-Rodriguez M, Medina JM: The role of connexin43-Src interaction in astrocytomas: a molecular puzzle. Neuroscience 2016, 323:183-194

71. Sandri M: Protein breakdown in muscle wasting: role of autophagylysosome and ubiquitin-proteasome. Int J Biochem Cell Biol 2013, 45:2121-2129

72. Rubinsztein DC: The roles of intracellular protein-degradation pathways in neurodegeneration. Nature 2006, 443:780-786

73. Ding G, Fu M, Qin Q, Lewis W, Kim HW, Fukai T, Bacanamwo M, Chen YE, Schneider MD, Mangelsdorf DJ, Evans RM, Yang Q: Cardiac peroxisome proliferator-activated receptor gamma is essential in protecting cardiomyocytes from oxidative damage. Cardiovasc Res 2007, 76:269-279

74. Okuno Y, Matsuda M, Miyata Y, Fukuhara A, Komuro R, Shimabukuro M, Shimomura I: Human catalase gene is regulated by peroxisome proliferator activated receptor-gamma through a response element distinct from that of mouse. Endocrinol Jpn 2010, 57: 303-309

75. Greer EL, Brunet A: FOXO transcription factors at the interface between longevity and tumor suppression. Oncogene 2005, 24: $7410-7425$

76. Ji L, Liu R, Zhang XD, Chen HL, Bai H, Wang X, Zhao HL, Liang X, Hai CX: N-acetylcysteine attenuates phosgene-induced acute lung injury via up-regulation of Nrf2 expression. Inhal Toxicol 2010, 22:535-542

77. Prescott LF, Park J, Ballantyne A, Adriaenssens P, Proudfoot AT: Treatment of paracetamol (acetaminophen) poisoning with N-acetylcysteine. Lancet 1977, 2:432-434 
78. Santos MM, Ohshima K, Pandolfo M: Frataxin deficiency enhances apoptosis in cells differentiating into neuroectoderm. Hum Mol Genet 2001, 10:1935-1944

79. Zhang L, Zhu Z, Liu J, Zhu Z, Hu Z: Protective effect of $\mathrm{N}$-acetylcysteine (NAC) on renal ischemia/reperfusion injury through Nrf2 signaling pathway. J Recept Signal Transduct Res 2014, 34:396-400

80. Karthikeyan G, Lewis LK, Resnick MA: The mitochondrial protein frataxin prevents nuclear damage. Hum Mol Genet 2002, 11: $1351-1362$

81. Richardson DR, Mouralian C, Ponka P, Becker E: Development of potential iron chelators for the treatment of Friedreich's ataxia: ligands that mobilize mitochondrial iron. Biochim Biophys Acta 2001, 1536:133-140

82. Lim CK, Kalinowski DS, Richardson DR: Protection against hydrogen peroxide-mediated cytotoxicity in Friedreich's ataxia fibroblasts using novel iron chelators of the 2-pyridylcarboxaldehyde isonicotinoyl hydrazone class. Mol Pharmacol 2008, 74: $225-235$
83. Johnson WM, Wilson-Delfosse AL, Mieyal JJ: Dysregulation of glutathione homeostasis in neurodegenerative diseases. Nutrients 2012, 4:1399-1440

84. Elbini Dhouib I, Jallouli M, Annabi A, Gharbi N, Elfazaa S, Lasram MM: A minireview on N-acetylcysteine: an old drug with new approaches. Life Sci 2016, 151:359-363

85. Kumar C, Igbaria A, D’Autreaux B, Planson AG, Junot C, Godat E, Bachhawat AK, Delaunay-Moisan A, Toledano MB: Glutathione revisited: a vital function in iron metabolism and ancillary role in thiol-redox control. EMBO J 2011, 30:2044-2056

86. Wang L, Ouyang B, Li Y, Feng Y, Jacquot JP, Rouhier N, Xia B: Glutathione regulates the transfer of iron-sulfur cluster from monothiol and dithiol glutaredoxins to apo ferredoxin. Protein Cell 2012, 3: 714-721

87. Yang X, Park S-H, Chang H-C, Shapiro JS, Vassilopoulos A, Sawicki KT, Chen C, Shang M, Burridge PW, Epting CL, Wilsbacher LD, Jenkitkasemwong S, Knutson M, Gius D, Ardehali H: Sirtuin 2 regulates cellular iron homeostasis via deacetylation of transcription factor NRF2. J Clin Invest 2017, 127:1505-1516 